Research Paper

\title{
Identification of low-dose radiation-induced exosomal circ- METRN and miR-4709-3p/GRB14/PDGFRa pathway as a key regulatory mechanism in Glioblastoma progression and radioresistance: Functional validation and clinical theranostic significance
}

\author{
Xinxin Wang1, Qinchen $\mathrm{CaO}^{2 \bowtie}$, Yonggang $\mathrm{Shi}^{2}$, Xiaolong $\mathrm{Wu}^{3}$, Yin $\mathrm{Mi}^{2}$, Ke $\mathrm{Liu}^{2}$, Quancheng Kan ${ }^{4}$, Ruitai \\ Fan ${ }^{5}$, Zhangsuo Liu 4 , Mingzhi Zhang ${ }^{3}$ \\ 1. Department of Neurology, The First Affiliated Hospital of Zhengzhou University, Zhengzhou 450052, People's Republic of China. \\ 2. Department of Radiation Therapy, The First Affiliated Hospital of Zhengzhou University, Zhengzhou 450052, People's Republic of China. \\ 3. Department of Medical Oncology, The First Affiliated Hospital of Zhengzhou University, Zhengzhou 450052, People's Republic of China. \\ 4. Department of Pharmacy and Internal Medicine, The First Affiliated Hospital of Zhengzhou University, Zhengzhou 450052, People's Republic of China. \\ 5. Department of Radiation Oncology, The First Affiliated Hospital of Zhengzhou University, Zhengzhou 450052, People's Republic of China.
}

$\triangle$ Corresponding author: Dr. Qinchen Cao, Department of Radiation Therapy, The First Affiliated Hospital of Zhengzhou University, Zhengzhou 450052, People's Republic of China. E-mail: fcccaoqs@zzu.edu.cn; Telephone: +86 15803878268.

(1) The author(s). This is an open access article distributed under the terms of the Creative Commons Attribution License (https://creativecommons.org/licenses/by/4.0/). See http://ivyspring.com/terms for full terms and conditions.

Received: 2020.12.18; Accepted: 2021.02.18; Published: 2021.03.02

\begin{abstract}
Glioblastoma is a central nervous malignancy with a very poor prognosis. This study attempted to explore the role of exosomes induced by low-dose radiation-induced (IdrEXOs) and IdrEXOs-derived circ-METRN in glioblastoma progression and radioresistance at the molecular, cellular, animal, and clinical levels. Results in the present study revealed that low-dose radiation stimulated the secretion of IdrEXOs which delivered high levels of circ-METRN. And circ-METRN-abundant IdrEXOs increased the expression of $\mathrm{Y}-\mathrm{H} 2 \mathrm{AX}$, indicating an efficient DNA damage-repair process in glioblastoma cells. The IdrEXOs-derived circ-METRN enhanced the glioblastoma progression and radioresistance via miR-4709-3p/GRB14/PDGFRa pathway. Up-regulating PDGFRa can rescue the tumor-promoting function of IdrEXOs in groups previously treated with inhibition of GRB14. Additionally, in-vivo experiments revealed that treatments with IdrEXOs promoted the growth of xenografted tumors and shortened the survival period. Furthermore, clinical researches indicated that circ-METRN may be transported into the bloodstream by exosomes in the early stages of fractionated radiotherapy. It has important clinical values to detect the serum exosomal circ-METRN in the early stage of radiotherapy, which is not only conducive to predict radioresistance and prognosis but also to assist MRI diagnosis in detecting the very early recurrence of glioblastoma. In summary, this study reveals for the first time that low-dose radiation-induced exosomal circ-METRN plays an oncogenic role in glioblastoma progression and radioresistance through miR-4709-3p/GRB14/PDGFRa pathway, providing mechanistic insights into the roles of circRNAs and a valuable marker for therapeutic targets in glioblastoma.
\end{abstract}

Key words: glioblastoma; low-dose radiation; exosome; circ-METRN; progression

\section{Introduction}

Glioblastoma is a malignant tumor of the central nervous system with a very high mortality rate [1]. The median survival time is less than 2 years [1]. Many clinical trials have been conducted to change therapy resistance and improve the survival of glioblastoma patients [2-5]. For instance, a convenient approach known as hypofractionated radiotherapy allows the total radiation dose to be converted into larger doses and fewer fractions, and then to be administered in a shorter period, although it was not 
documented to significantly improve overall survival of glioblastoma patients [5]. Despite major advances in radiation technology, the overall outcome of radiotherapy in glioblastoma remains far from optimal, as tumors were identified to be inherently radioresistant and to develop increased radioresistance, especially upon recurrence [2-4]. The shorter survival period and the biological resistance to radiotherapy make the role of radiotherapy divergent in glioblastoma $[2,3]$. It is, thus, essential to select glioblastoma patients who respond well to postoperative chemoradiotherapy and to explore individualized treatment protocols including optimal timing, fractionation, dose, and radiotherapy techniques. Especially for MGMT-unmethylated patients, it is very urgent to find effective indicators and valuable biomarkers to guide more aggressive and effective individualized multimodality therapies.

More importantly, many patients with glioblastoma may suffer undetectable recurrence in the early stages of radiotherapy [4, 6, 7]. This very early recurrence is manifested on magnetic resonance imaging (MRI) as a slight abnormal signal, which is difficult to distinguish from cerebral edema, postoperative inflammation, and pseudoprogression $[4,6]$. Many studies have deeply analyzed the causes of recurrence and found that very early recurrence, insufficient radiotherapy, or interruption of radiotherapy may instead accelerate the progression and promote radioresistance of glioblastoma cells [2-5]. Meanwhile, basic laboratory studies have also confirmed that high-grade gliomas are very insensitive to low-dose radiotherapy, and radioresistance is very common in glioblastoma [8-10]. Berg et al. demonstrated that all cell lines, including highgrade astrocytoma U-251MG cell lines, developed radioresistance within 2-3 weeks during fractionated radiotherapy [7]. Unexpectedly, research on other malignant tumors found that low-dose radiation can promote tumor progression [11, 12].

These earlier studies prompted us to further identify the molecular expression patterns in glioblastoma treated with different radiation doses. Many factors, including bystander effects, exosomes and circular RNAs (circRNAs), serve important roles in the progression, radioresistance, and recurrence of glioblastoma [13, 14]. Thereinto, exosomes are nano-sized membrane vesicles with diameters of 30 $\mathrm{nm}$ to $100 \mathrm{~nm}$ [15]. It has previously been documented that exosomes exert a key role in regulating cancer cell-cell communication, tumor-stromal interactions, activation of signaling pathways, and immunomodulation through effectively delivering pathogenic components, such as proteins, RNAs, DNA fragments, and lipids [15]. In malignant tumors such as pancreatic cancer, liver cancer, stomach cancer, colon cancer, breast cancer and prostate cancer, tumor cells are very active in secreting exosomes [16-21]. As an effective carrier, exosomes can transport various molecular components [16-21]. This in turn affects the progression and therapeutic resistance of tumor cells themselves or adjacent cells [16-21]. However, few studies identified the functions of exosomes in glioblastoma progression and radiotherapy [22, 23]. Emerging data suggest that different doses of radiation can not only promote the production and transport of exosomes but also enhance the absorption of exosomes by other cells [24-26]. Radiation-induced exosomes increase the tumor burden, reduce survival, induce bystander effects, and promote radiotherapy resistance [15]. This allows exosomes to be used as biomarkers to monitor the effects of radiotherapy and predict the prognosis of glioblastoma patients [15]. However, the content and component carried by low-dose radiation-induced exosomes (ldrEXOs) remain unclear. There are still some controversies about the regulatory effect of exosomes on radiotherapy resistance and its prognostic value [27, 28].

Early studies indicated that circRNAs played regulatory roles mainly in cells after production [14]. Few studies have shown that circRNAs can be transported out of the cell to affect the cellular microenvironment, radioresistance, and bystander effect. A few studies have shown that circRNAs can enhance the radioresistance of malignant tumors [29], but some other studies state that circRNAs can reduce the radioresistance of malignant tumors [16, 29-31]. It is obvious that there are still some controversies about the effect pattern of circRNAs on progression and radioresistance of malignant tumor [16, 29-31]. Recent studies have revealed that circRNAs are abundant in exosomes [16, 31]. Exosomal circRNAs play important roles in the progression of other malignant tumors while the effect of circ-METRN on glioblastoma radiotherapy resistance remains unknown $[16,31]$.

In this study, we reported for the first time that the identification of circ-MTRN in low-dose radiation-induced exosomes (ldrEXOs), and further explored its functions and underlying mechanisms in glioblastoma progression and radioresistance at molecular, cellular, animal and clinical levels. We identified the role of low-dose radiation-induced exosomal circ-METRN and miR-4709-3p/GRB14/ PDGFRa pathway, bringing a novel insight into the investigation of exosomal circRNAs and providing potential targets for anti-glioblastoma therapy. 


\section{Methods}

\section{Cell culture}

Human SW1783 and U-118MG glioblastoma cell lines were purchased from the Cell Resource Center of the Shanghai Institute of Biological Sciences. Normal human astrocytes (NHA) were purchased primarily from Sciencell Research Laboratories (Carlsbad, CA, USA) as well. Glioblastoma cells cultured in DMEM medium (Gibco, USA) containing 10\% fetal calf serum at $37{ }^{\circ} \mathrm{C}$ were placed in a humidified atmosphere containing $5 \% \mathrm{CO}_{2}$. The cell lines of the same batch were stably subcultured for five generations.

\section{Exosome isolation and co-culture}

To isolate exosomes, glioblastoma cells (SW1783 and U-118MG) previously treated with low-dose radiation (LDR) or high-dose radiation (HDR), were cultured for $48 \mathrm{~h}$ and the supernatant was collected. To deplete them of the cells and fragments, the supernatants were then centrifuged twice $(1,000 \times \mathrm{g}$ for $10 \mathrm{~min}$ and $3,000 \times \mathrm{g}$ for $30 \mathrm{~min}$ at $4^{\circ} \mathrm{C}$ ). Total Exosome Isolation Reagent (Thermo Fisher Scientific, Inc.) was added overnight, then followed by centrifugation $10,000 \times \mathrm{g}$ for $1 \mathrm{~h}$ at $4{ }^{\circ} \mathrm{C}$. Exosomes were resuspended in PBS and stored at a temperature of $-80{ }^{\circ} \mathrm{C}$. Exosomes derived from cells in LDR groups (ldrEXO) were added to $10^{5}$ glioblastoma cells at a concentration of $50 \mathrm{ng} / \mathrm{ml}$ serum-free DMEM for $24 \mathrm{~h}$. LDR cells were then treated with knockdown of circ-METRN before collection of exosomes (ldrEXO_ siCIRC) in the supernatant.

\section{Transmission electron microscopy}

We added the exosome suspension to an equal volume of $4 \%$ paraformaldehyde (Nacalai Tesque, Inc., Kyoto, Japan), and applied the mixture to a Formvar/carbon film-coated transmission electron microscope (TEM) grid (Alliance Biosystems, Inc., Osaka, Japan). Subsequently, the sample was fixed by incubation with $1 \%$ glutaraldehyde for $5 \mathrm{~min}$, washed with PBS, and incubated with 1\% uranyl acetate for 5 min. The sample was then observed using a TEM (Hitachi H7500; Hitachi, Ltd., Tokyo, Japan).

\section{Nanoparticle tracking analysis}

Nanoparticle tracking analysis (NTA) (NanoSight NS300, Malvern Instruments, UK) was applied for size distribution and concentration measurements of exosomes in liquid suspension from the properties of both light scattering and Brownian motion. To detect nanovesicles, the NanoSight NS300 with a $405 \mathrm{~nm}$ laser instrument was used. For each sample, five video times of 60 seconds were taken. Data were analyzed using the NTA 3.0 software, and the Hydrodynamic diameters of each particle were calculated using the Stokes-Einstein equation as following: $\mathrm{D}=\mathrm{kT} /(6 \Pi \eta \mathrm{r})$. [D is the diffusion coefficient, $\mathrm{k}$ is Boltzmann's constant; $\mathrm{T}$ is the absolute temperature; $r$ is the radius of the particle; $\eta$ is the viscosity of the fluid, which means a spherical particle moving with the uniform velocity in a continuous fluid].

\section{Microarray analysis and bioinformatics analysis}

CircRNAs were enriched by removing linear RNAs with Rnase R (Epicentre, Madison, WI, USA). Arraystar Human circRNA Array and LncPath Human Cancer Array were used for hybridization, and then the Agilent Scanner G2505C (Jamul, CA, USA) was used for scanning. Heat maps were developed using HemI1.0.1 software (http:// hemi.biocuckoo.org/down.php). And then, the downstream signaling pathways of circ-METRN were also analyzed.

\section{Fluorescence in situ hybridization (FISH)}

Specific probes to the sequences of circ-METRN (green-labeled, Biosense, Guangzhou, China) (5'GGCATGGGAGATAGGGAGAC -3') and miR4709-3p probe (red-labeled, Exiqon, Copenhagen, Denmark) were used in situ hybridization. Nuclei were counted by staining with 4, 6-diamidino-2phenylindole (DAPI). All procedures were conducted according to the manufacturer's instructions (Genepharma, Shanghai, China). All images were acquired on a Zeiss LSM880 NLO (2+1 with BIG) confocal microscope system (Leica Microsystems, Mannheim, Germany).

\section{Quantitative real-time PCR (RT-PCR) and RNase R treatment}

RT-PCR was performed as previously described [14]. The primers used for RT-PCR can be found in Supplementary Material.

Transcription was prevented by the addition of 2 $\mathrm{mg} / \mathrm{mL}$ Actinomycin D or DMSO (Sigma-Aldrich, St. Louis, MO, USA) as a negative control (NC). Total RNA $(2 \mu \mathrm{g})$ was incubated for $30 \mathrm{~min}$ at $37^{\circ} \mathrm{C}$ with 3 $\mathrm{U} / \mu \mathrm{g}$ of RNase R (Epicentre, Madison, WI, USA). After treatment with RNase R, the RNA expression levels of linear-METRN and circ-METRN were detected by RT-PCR.

\section{RNA interference and transfection assays}

Small interfering RNAs (siRNAs) targeting linear transcript or targeting the backsplice sequence of circRNA were designed and synthesized by RiboBio (Guangzhou, China). RNA interference and transfection assays were performed as previously 
described [14]. More details about the siRNAs used in this study can be found in Supplementary Material.

\section{MTT assays and cell apoptosis assays and colony formation assay}

At the indicated time point after transfection, cells were collected by centrifugation after incubation with $5.0 \mathrm{mg} / \mathrm{ml} \quad 3$-(4,5-dimethyl-2-thiazolyl)-2,5diphenyl-2-H-tetrazolium bromide (MTT) Dimethyl sulfoxide $(200 \mu \mathrm{l})$ was added into the sediments, and then spectrophotometry at $490 \mathrm{~nm}$ was used for the measurement of the absorbance. To identify cell apoptosis, cells were also stained using an annexin $\mathrm{V}$ apoptosis kit (eBiosciences, USA) and analyzed using flow cytometry.

For colony formation capacity detection, glioblastoma cells were plated in six-well plates in triplicate (400 cells/well). The cells were incubated for 2 weeks, and the culture media was refreshed per $3 \mathrm{~d}$. The colonies were stained with crystal violet. The colonies including 50 cells or more cells were counted.

\section{Wound healing assays and transwell assays}

Wound healing assays and transwell assays were performed as previously described [14]. Transwell inserts (Corning, NY, USA) and Matrigel (BD Biosciences, CA, USA) were purchased. An inverted microscope (Motic Instruments, Richmond, BC, Canada) was applied in capturing images.

\section{Luciferase reporter assays}

Human embryonic kidney (HEK) 293T cell lines were purchased from the Cell Resource Center of the Shanghai Institute of Biological Sciences. Luciferase reporter assays were performed as previously described [14].

\section{Western blot analysis}

Western blot was performed as previously described [14]. More details about antibodies can be found in Supplementary Material.

\section{Orthotopic xenograft model}

SW1783 and U-118MG cells were injected $\left(5 \times 10^{7}\right.$ cells $/ \mathrm{ml}$ ) into the right striatum through a burr hole in the skull of BALB/C nude mice (4-6 weeks old, 18-22 $\mathrm{g}$, six female mice per group) using a $10-\mu 1$ Hamilton syringe. Before inoculation, mice were anesthetized with an intraperitoneal injection of $0.1 \%$ pentobarbital $(0.1 \mathrm{ml})$ in an animal stereotaxic apparatus. After inoculation, the wound was sutured to avoid infection. Tumor growth was monitored and measured via bioluminescence imaging in vivo by the injection of $10 \mu \mathrm{l} / \mathrm{g}$ bodyweight of D-luciferin using the IVIS Spectrum system (Perkin Elmer, Waltham, USA) according to the recommended procedure.
Animals were daily monitored for cachexia (evaluated by bodyweight waste). Animals that lost about $20 \%$ of the bodyweight were euthanized. All animals' experimental protocols were approved by the Ethics Committee of Zhengzhou University and the Experimental Animal Center of Zhengzhou University for compliance with the National Institutes of Health for use of laboratory animals or equivalent. All methods were carried out following the relevant guidelines and regulations. Every effort was made to minimize the number of animals used and their suffering.

\section{Irradiation procedure}

Exponentially growing cells were seeded in culture flasks, $72 \mathrm{~h}$ before the first experimental irradiation, and subsequently exposed to a variety of single doses: $2 \mathrm{~Gy}$ (approximately the daily dose used in humans), 4 Gy, $6 \mathrm{~Gy}, 8 \mathrm{~Gy}$, and $10 \mathrm{~Gy}$ of the X-ray beam in a linear accelerator (Elekta Synergy, Sweden). Six of the seeded plates served as controls and received no irradiation. The medium was refreshed twice every week. Cell counts were performed using a Coulter Counter (Beck-man Coulter, Inc., Fullerton, USA).

Mice were locally irradiated at a single dose of 10Gy under anesthesia. Control animals were anesthetized and sham irradiated. Mice that presented neurological symptoms (i.e. hydrocephalus, seizures, inactivity, and/or ataxia) or moribund were also sacrificed.

\section{Y-H2AX staining}

Cells derived from glioblastoma biopsy specimens, treated with ldrEXO or ldrEXO_siCIRC were exposed to a radiation dose of 10Gy. Six hours or $24 \mathrm{~h}$ post-radiation, cells were fixed, and intracellular staining with an Alexa Fluor 488 anti-H2AXPhosphorylated (Ser139) antibody was performed according to the manufacturer's protocol (BioLegend). Stained cells were analyzed by the BD Accuri C6 cytometer and the data were analyzed by the $\mathrm{BD}$ Accuri C6 software.

\section{Clinical research and human specimens}

From January 1, 2015 to December 31, 2019, at the First Affiliated Hospital of Zhengzhou University, glioblastoma patients who underwent surgical resections were reviewed. The pathological classification was based on the WHO 2007 classification of tumors by two experienced neuropathologists. The inclusion and exclusion criteria were shown in Supplementary Material. Disease-free survival (DFS) was calculated from the date of complete resection to any recurrent disease, distant metastasis, or death. Overall survival (OS) was 
calculated from the date of surgery to the date of death from any cause.

Fresh frozen pathological specimens and serum specimens were both obtained. Serum specimens were acquired from patients before operation, before radiotherapy, during radiotherapy (in the first week of radiotherapy), or after radiotherapy, respectively. The circ-METRN levels in glioblastoma tissues and serum exosomes were analyzed and compared. To compare the molecular expression differences among gliomas of different grades ( $\mathrm{n}=12$, each group), some other specimens, including peritumoral normal brain tissues (PNBTs) and Grade I-III gliomas, were also collected. PNBTs were used as NCs. All experimental protocols in this section were approved by the Ethics Committee of Zhengzhou University. Written informed consent was signed and obtained from all individual participants or their legal guardians in this study.

\section{Statistical analysis}

All statistical tests were two-sided. Experimental data are shown as the mean \pm standard deviation (SD). Error bars were obtained according to the standard deviation. Student's two-tailed unpaired $t$ test was used to determine the statistical significance. And a $\mathrm{P}$ value $<0.05$ was considered statistically significant. The Kaplan-Meier method and log-rank test were used to compare survival rates. The multivariate survival analysis was applied to identify independent prognostic factors. Statistical analyses were performed using SPSS software version 17.0 (SPSS Inc., Chicago, IL, USA) and R 2.8.0 statistical package (the $\mathrm{R}$ Core Team, Vienna, Austria).

\section{Results}

LDR stimulated the secretion of glioblastoma exosomes that are enriched with high levels of circ-METRN, and circ-METRN-abundant IdrEXOs may modulate DNA damage response

To identify and analyze exosomes induced by different doses of irradiation, electron microscope scanning and NAT analysis were both performed (Figure 1A-B). Expressions of CD63 and TSG101 proteins were also detected by Western blot (Figure 1C). After microarray analysis for ldrEXOs, a total of 23 circRNAs, including circ-METRN, were identified as abnormally expressed in glioblastoma exosomes (Figure 1D-E). The expression of exosomal circ-METRN in the LDR group was significantly higher than that in the NC group and HDR group (Figure 1F). In groups treated with ldrEXOs, levels of
circ-METRN in exosomes and cell lysates were both increased (Figure 1F). The relative expression levels of circ-METRN and its cognate linear mRNA before and after treatment with Rnase $\mathrm{R}$ were also analyzed in NHA and glioblastoma cell lysates (Figure 1G-H).

Ionizing radiation is known to induce DNA damages leading to lethal cytotoxicity, and high activation of the DNA damage repair is an important component of radioresistance of glioblastoma in particular. To determine whether inhibition of exosomal circ-METRN affected radiation-induced phosphorylation of $\mathrm{H} 2 \mathrm{AX}(\gamma-\mathrm{H} 2 \mathrm{AX})$, a marker of DNA breaks, we further examined the expression of $\gamma-\mathrm{H} 2 \mathrm{AX}$. As expected, $\mathrm{\gamma}-\mathrm{H} 2 \mathrm{AX}$ staining increased rapidly following radiation and returned to basal level at $24 \mathrm{~h}$ in cells treated with ldrEXOs (Figure 1I), indicating an efficient DNA damage-repair process in glioblastoma cells. However, ldrEXOs with inhibition of circ-METRN (ldrEXOs_siCIRC) did not significantly exhibit the promoting effect, suggesting the important role of exosomal circ-METRN in the DNA damage-repair process in glioblastoma cells.

\section{Circ-METRN-abundant IdrEXOs promoted progression and radioresistance of glioblastoma cell lines}

Compared with the NC group (a), ldrEXOs with high levels of circ-METRN $(\boldsymbol{\delta}, \boldsymbol{\varepsilon})$ promoted proliferation, radioresistance, apoptosis resistance, invasion, and migration abilities of glioblastoma cell lines while ldrEXOs with inhibition of circ-METRN (ldrEXOs_siCIRC) $(\boldsymbol{\beta}, \zeta)$ did not significantly exhibit the promoting effect (Figure 2A-E). ldrEXOs with high levels of circ-METRN, instead of

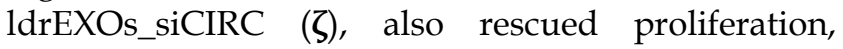
radioresistance, apoptosis resistance, invasion, and migration abilities of glioblastoma cell lines previously treated with high-dose radiation (HDR) $(\varepsilon)$ (Figure 2A-E).

\section{Exosomal circ-METRN was efficiently transported into glioblastoma cells by IdrEXOs and acts as a miR-4709-3p sponge}

To verify whether circ-METRN can be effectively transported by ldrEXOs among glioblastoma cells, circ-METRN-knockdown (siCIRC) cells of glioblastoma were treated with ldrEXOs. As a result, the level of circ-METRN in these cells was significantly improved (Figure 3A). Then, the expression level of circ-METRN in different grades of glioma tissues was proved to be very different (Figure 3B). What's more, circ-METRN was predicted to share miRNA response elements (MREs) of miR-4709-3p (Figure 3C). Further luciferase reporter assays confirmed that circ-METRN had a sponging effect on 
miR-4709-3p (Figure 3D). Circ-METRN can affect the expression levels of miR-4709-3p (Figure 3E) while
miR-4709-3p did not impact the expression of circ-METRN (Figure 3F).
A

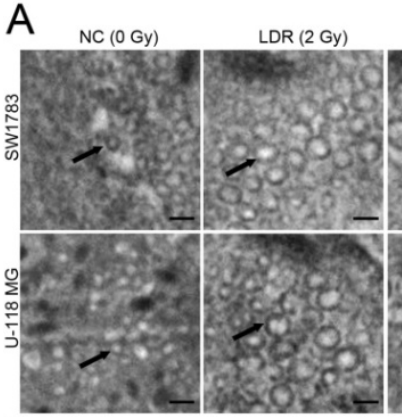

D

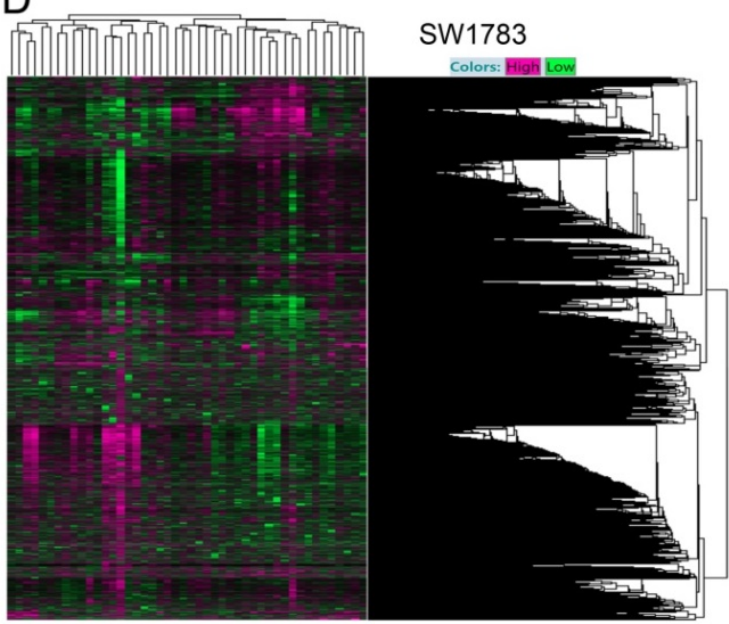

E

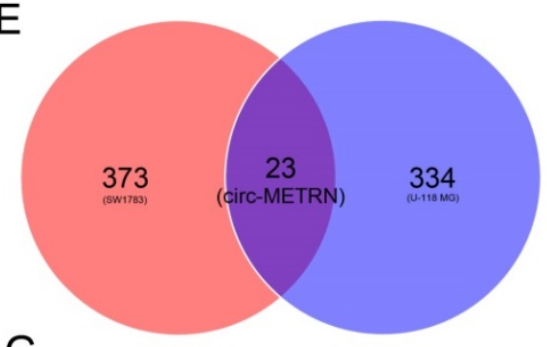

G
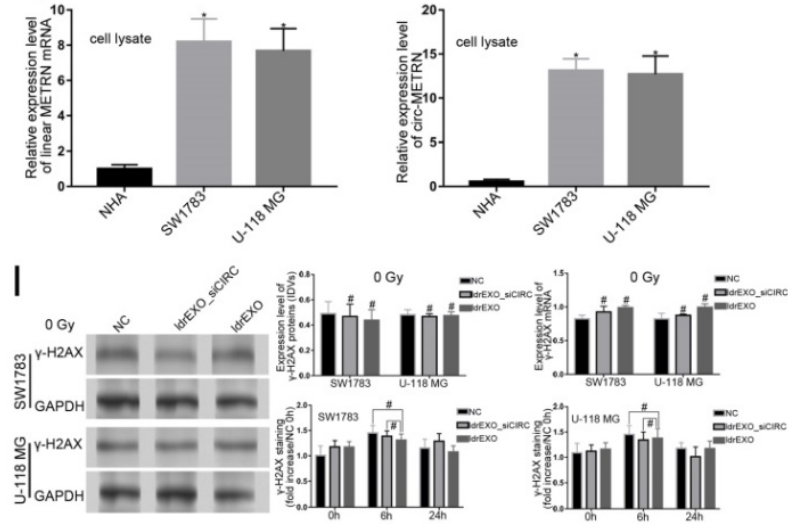

B

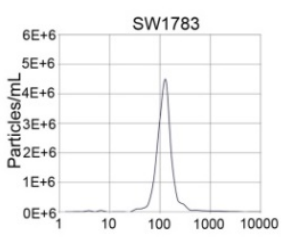

C

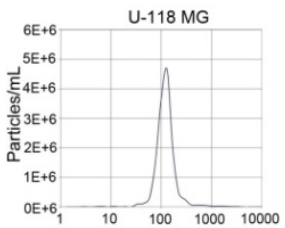

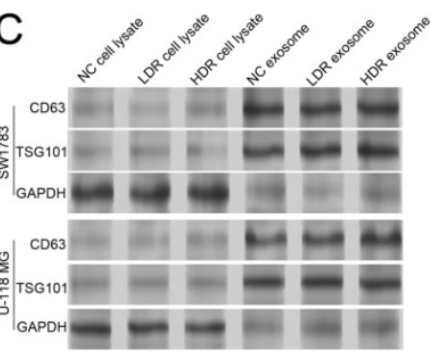

U-118 MG

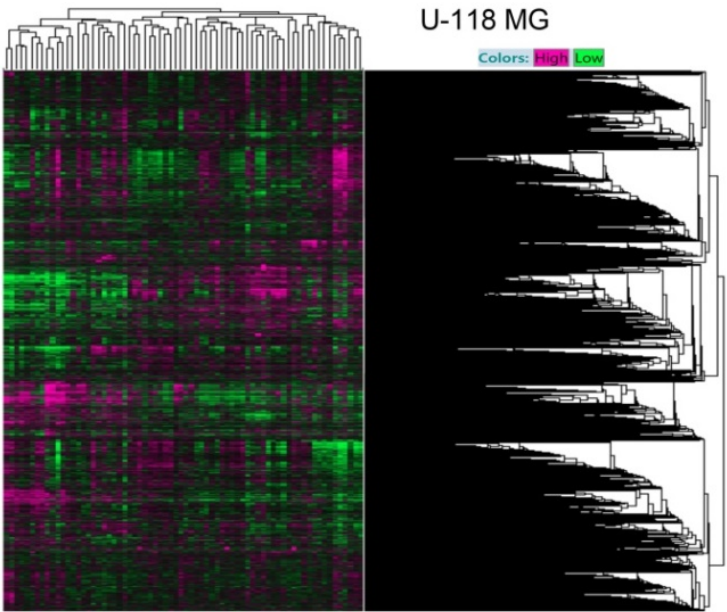

$\mathrm{F}$
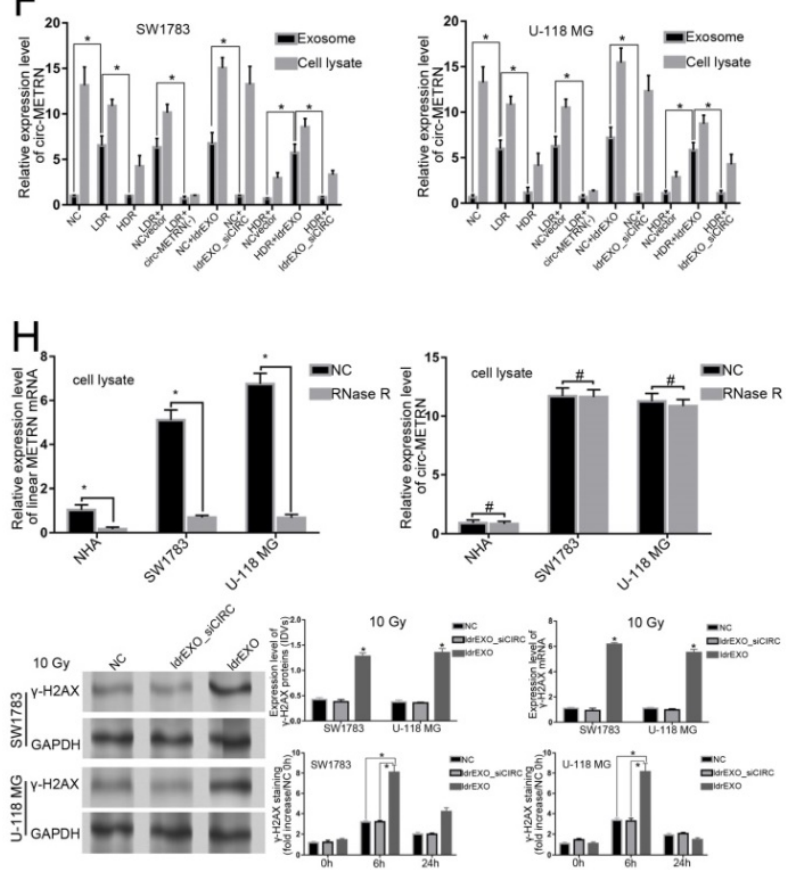

Figure 1. Characterization of exosomes derived from glioblastoma cell lines treated with low-dose radiation. A. Electron microscope scanning of exosomes (arrows) isolated from SW1783 and U-118 MG cell lines ( $\mathrm{n=6}$ ) (scale bar: $50 \mathrm{~nm}$ ). B. The size range of the low-dose radiation-induced exosomes (IdrEXOs) isolated from SW1783 and U-118 MG cell lines checked by NAT analysis. C. Western blot analysis of exosomal markers, including CD63 and TSG101 ( $=6$ ). D. Heat maps of the expression profile consisting of three pairs of exosomes in SW1783 and U-118 MG cells treated with low-dose radiation (LDR) and control cells (NC) by RNA sequencing (23 pairs). E. A Venn diagram of the intersection between results of the differential expression of SW1783 and U-118 MG cells. F. RT-PCR analyses of the content of exosomal circ-METRN in different groups $(n=6)$. G. The relative expression levels of circ-METRN and its cognate linear mRNA in normal human astrocytes $(N H A)$ and glioblastoma cell lines $(n=6)$. $\mathbf{H}$. Expression levels of circ-METRN and its cognate linear mRNA in glioblastoma cell lines after treatment with Rnase R. I. IdrEXOs increased the expression of $\mathrm{Y}$ - $\mathrm{H} 2 \mathrm{AX}$, indicating an efficient DNA damage-repair process in glioblastoma cells $(n=6)$. IdrEXOs with inhibition of circ-METRN (IdrEXOs_siCIRC) did not significantly exhibit the promoting effect, indicating the important role of exosomal circ-METRN in the DNA damage-repair process in glioblastoma cells $(n=6)$. $* P<0.05, \# P>0.05$. 

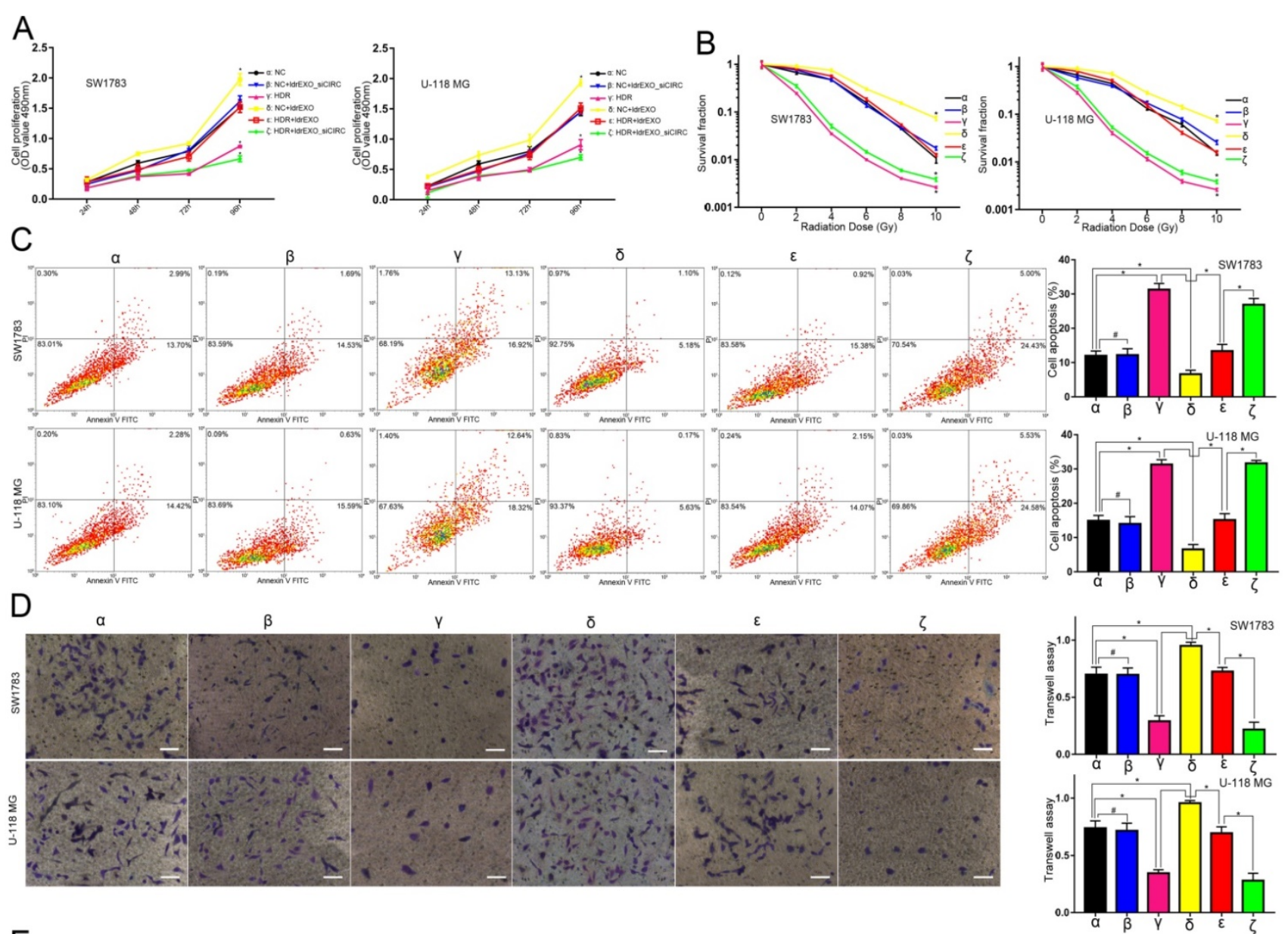

$\mathrm{E}$
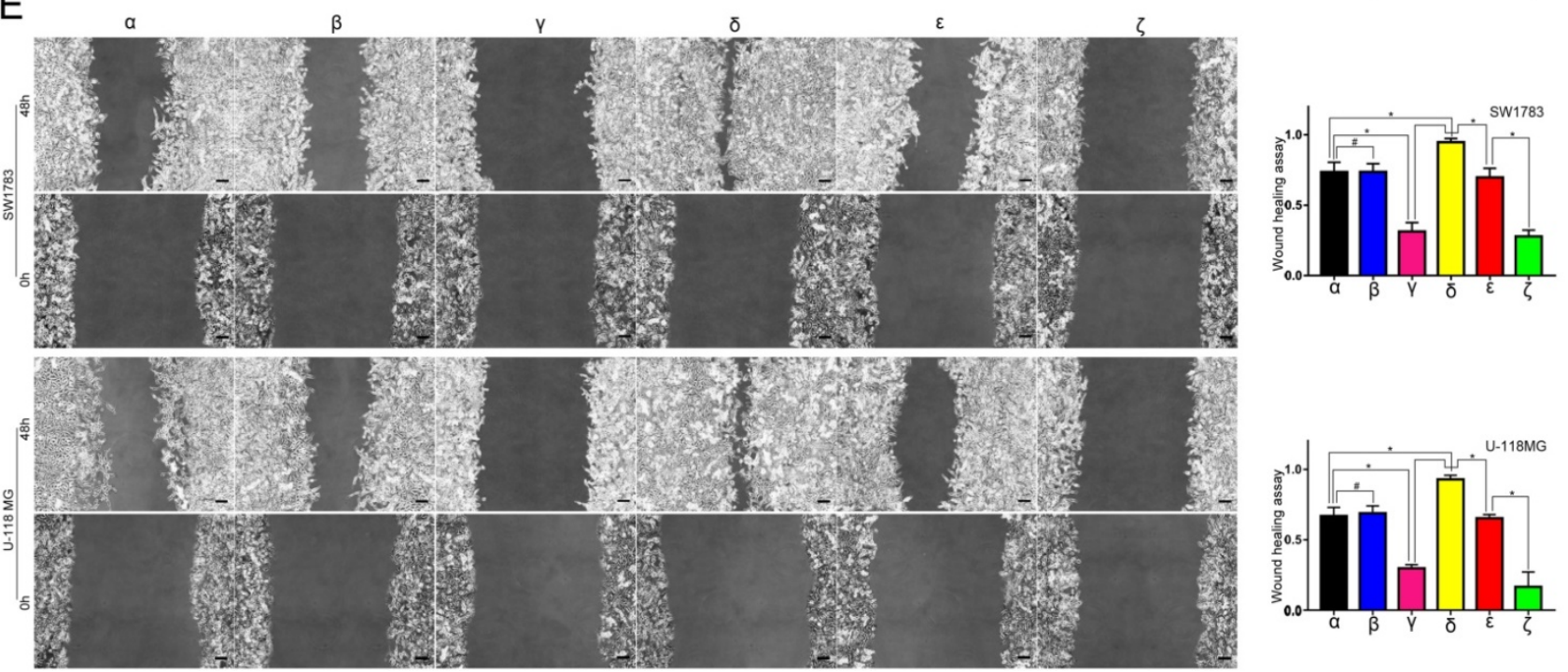

Figure 2. circ-METRN-abundant IdrEXOs promote progression and radioresistance of glioblastoma cell lines. A. Cell proliferation assays were conducted to analyze the effects of circ-METRN-abundant IdrEXOs on glioblastoma cell proliferation ability $(\boldsymbol{\delta}, \boldsymbol{\varepsilon})$. IdrEXOs_siCIRC: IdrEXOs with inhibition of circ-METRN ( $\boldsymbol{\beta}, \zeta)$. B. Radiation sensitivity was tested with colony formation assays in both cell lines. $\mathbf{C}$. Apoptosis resistance was assessed by flow cytometry with annexin $\mathrm{V}$-FITC/propidium iodide (PI). D-E. Transwell assays and wound healing assays were used to analyze glioblastoma cell migration and invasion ability, respectively. $* P<0.05$, $\# P>0.05$.

The miR-4709-3p expression was significantly affected by the IdrEXO-transported circMETRN and GRB 14 is a direct target of miR-4709-3p

Without the treatment of miR-4709-3p mimics,
miR-4709-3p was under-expressed in glioblastoma cells and tissues (Figure 4A-C). Despite the low expression of miR-4709-3p in glioblastoma, the sponging effect of the ldrEXO-transported circ-METRN was still observed (Figure 4A). To verify whether exosomal circ-METRN can still play a 
sponging role in the high level of miR-4709-3p, we also observed the effect of ldrEXO treatment on the miR-4709-3p level in glioblastoma cells treated with the previous transfection of miR-4709-3p mimics (Figure 4A). Results revealed that the level of miR4709-3p in these cells was also significantly decreased after treatment with ldrEXOs (Figure 4A). Moreover, GRB14 was then predicted as a direct target of miR-4709-3p (Figure 4D). Further luciferase reporter assays confirmed that miR-4709-3p had a targeting relationship to GRB14 (Figure 4E). And miR-4709-3p can affect the expression levels of GRB14 mRNA and protein (Figure 4F).

\section{Low-dose radiation-induced exosomal circ-METRN promotes glioblastoma cell progression and radioresistance by regulating the miR-4709-3p/GRB 14 axis}

ldrEXOs promoted proliferation, radioresistance, apoptosis radioresistance, invasion, and migration
A NHA
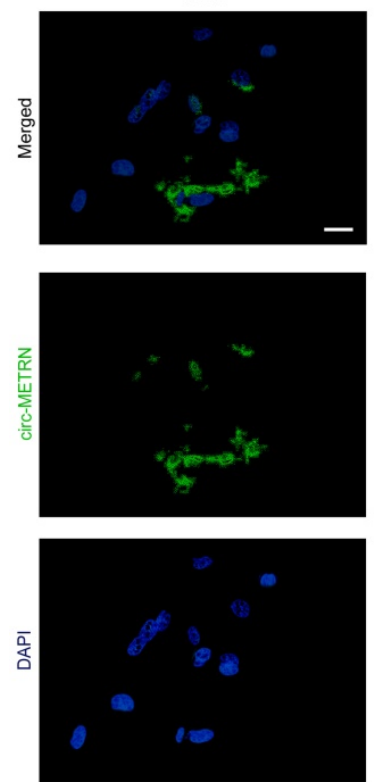

B

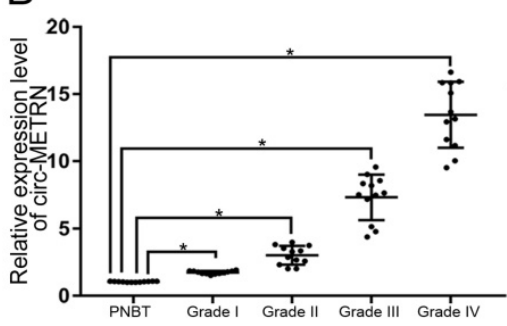

SW1783 (siCIRC)
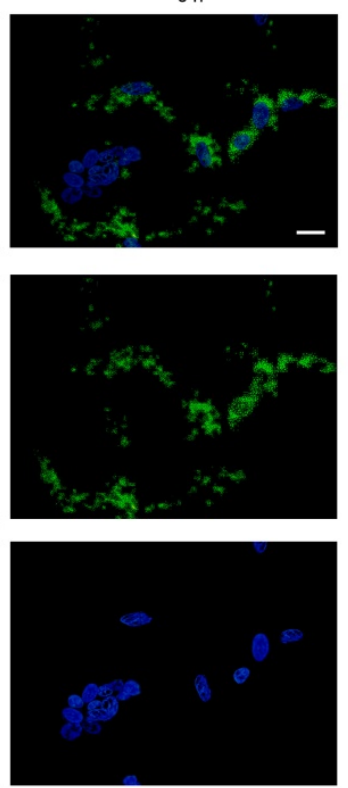
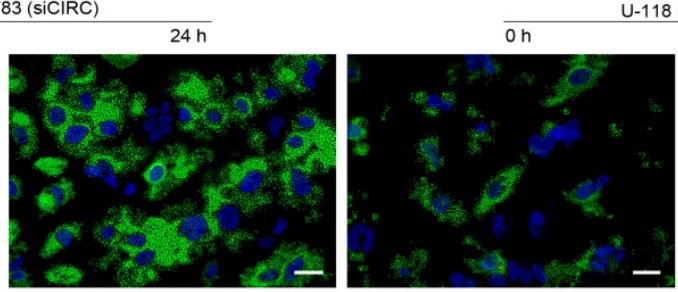

-118 MG (SICIRC)
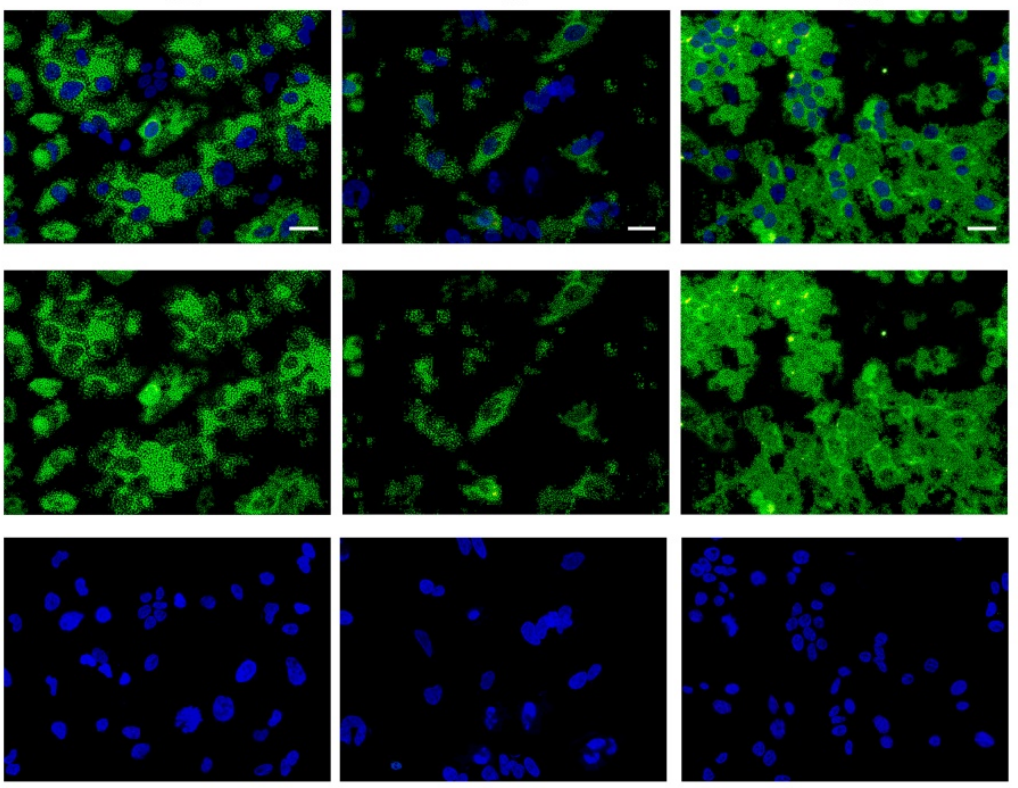

C
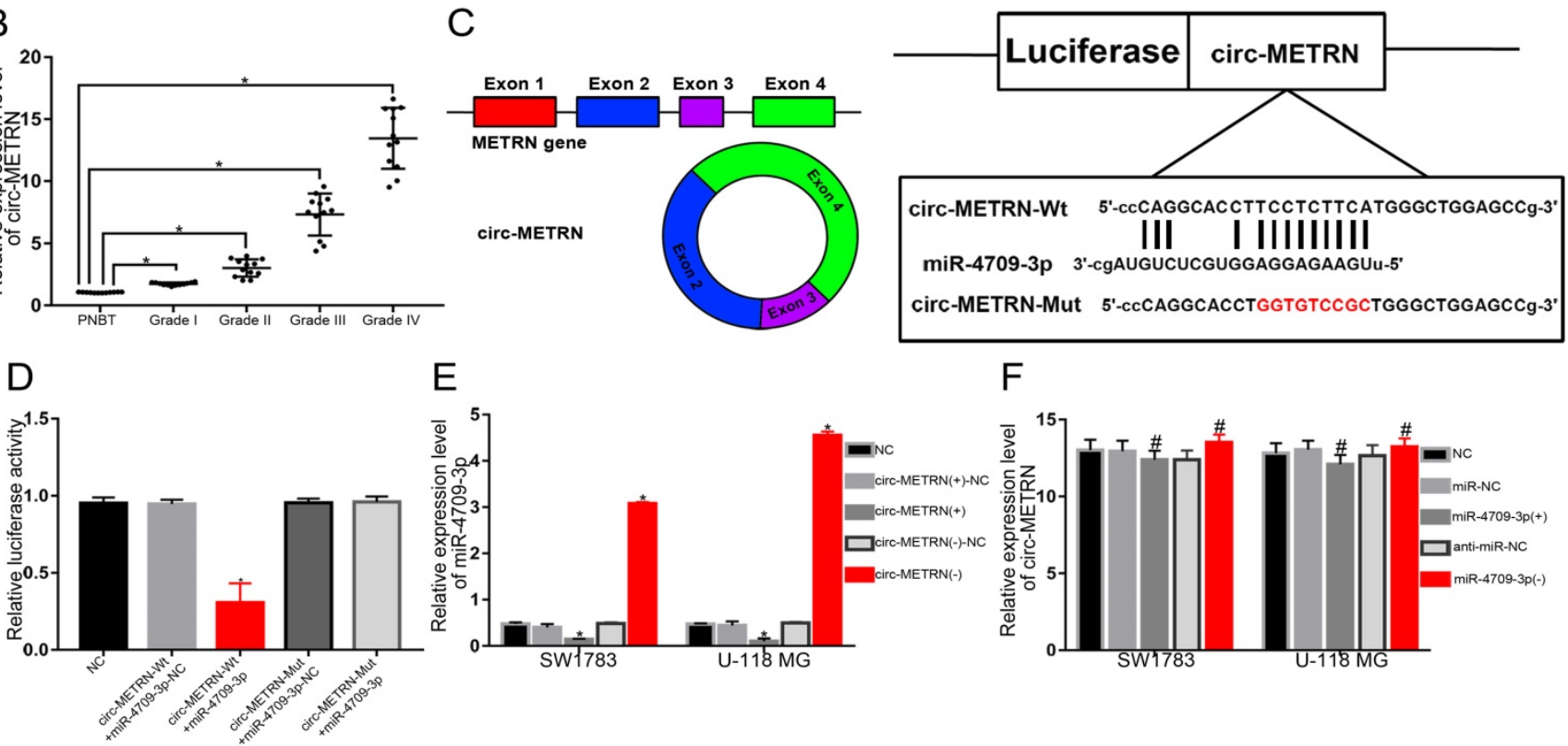

$\mathrm{F}$

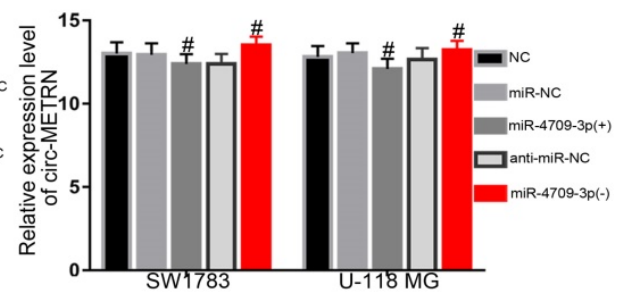

Figure 3. Exosomal circ-METRN was efficiently transported into glioblastoma cells by IdrEXOs and acts as a miR-4709-3p sponge. A. After treatments with IdrEXOs, the levels of circ-METRN in circ-METRN-knockdown $($ siCIRC) glioblastoma cells were significantly improved $(\mathrm{n}=6)(\mathrm{scale}$ bar: $20 \mu \mathrm{m})$. B. Expression levels of circ-METRN in PNBT and glioblastoma $(n=12)$. C. The cartoon of circ-METRN arose from the METRN gene and the predicted miR-4709-3p binding sites in circ-METRN (Wt) and the designed mutant sequences (Mut). D. Luciferase reporter assay of HEK 293T cells with different co-transfection treatments. E. RT-PCR analysis showed that circ-METRN regulated miR-4709-3p expression in the circ-METRN(+) group and the circ-METRN(-) group, respectively. F. RT-PCR analysis further verified that miR-4709-3p did not significantly attenuate circ-METRN expression either in the miR-4709-3p $(+)$ group or in the miR-4709-3p(-) group. $* P<0.05, \# P>0.05$. 
abilities of glioblastoma cell lines $(\boldsymbol{\beta})$ while knockdown of circ-METRN ( $\gamma$ ) or up-regulation of miR-4709-3p (ס) attenuated these abilities of glioblastoma cell lines previously treated with
ldrEXOs (Figure 5A-E). Knockdown of miR-4709-3p $(\varepsilon)$ or up-regulation of GRB14 $(\zeta)$ rescued progression and radioresistance of glioblastoma cell lines (Figure 5A-E).
A
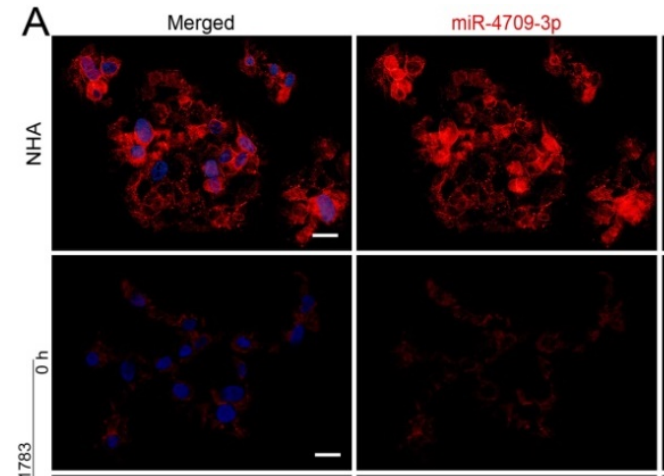

$\sum_{\omega}^{\infty}$
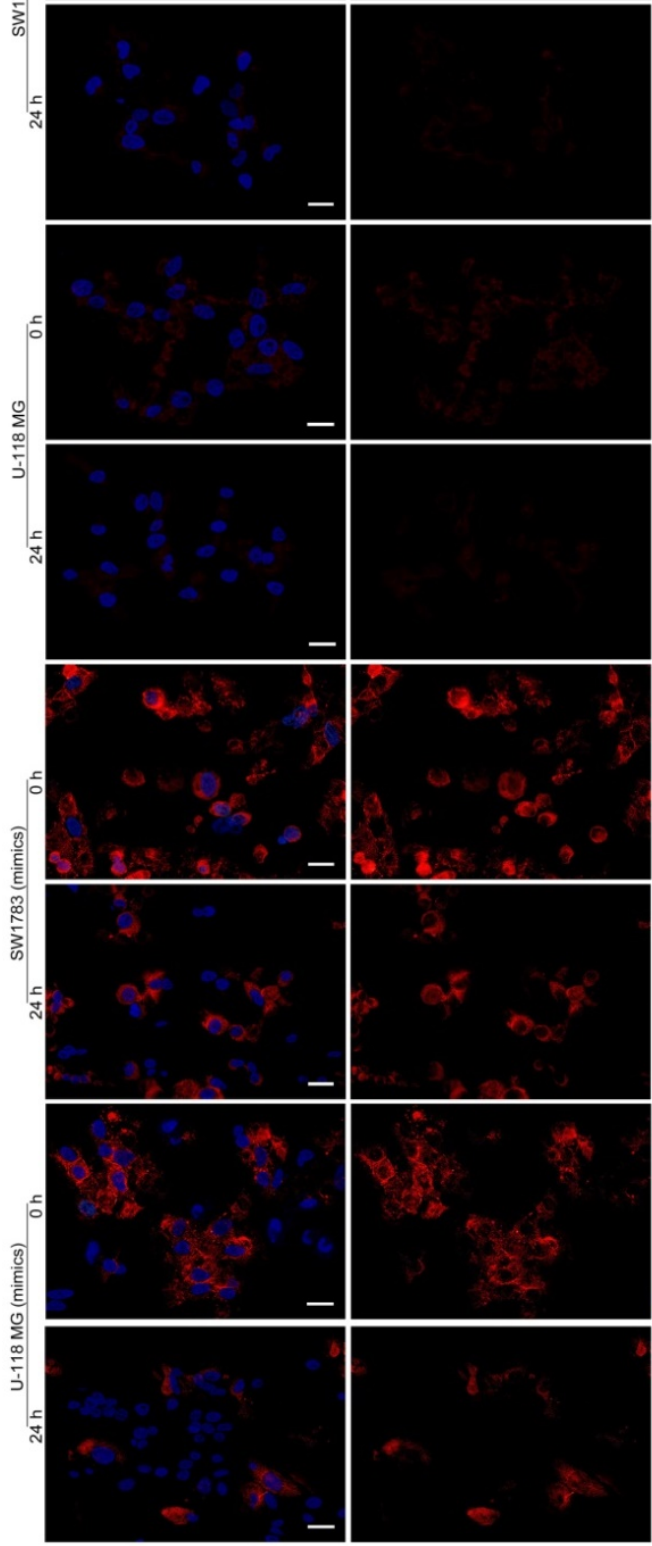

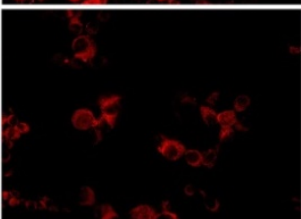

miR-4709-3p
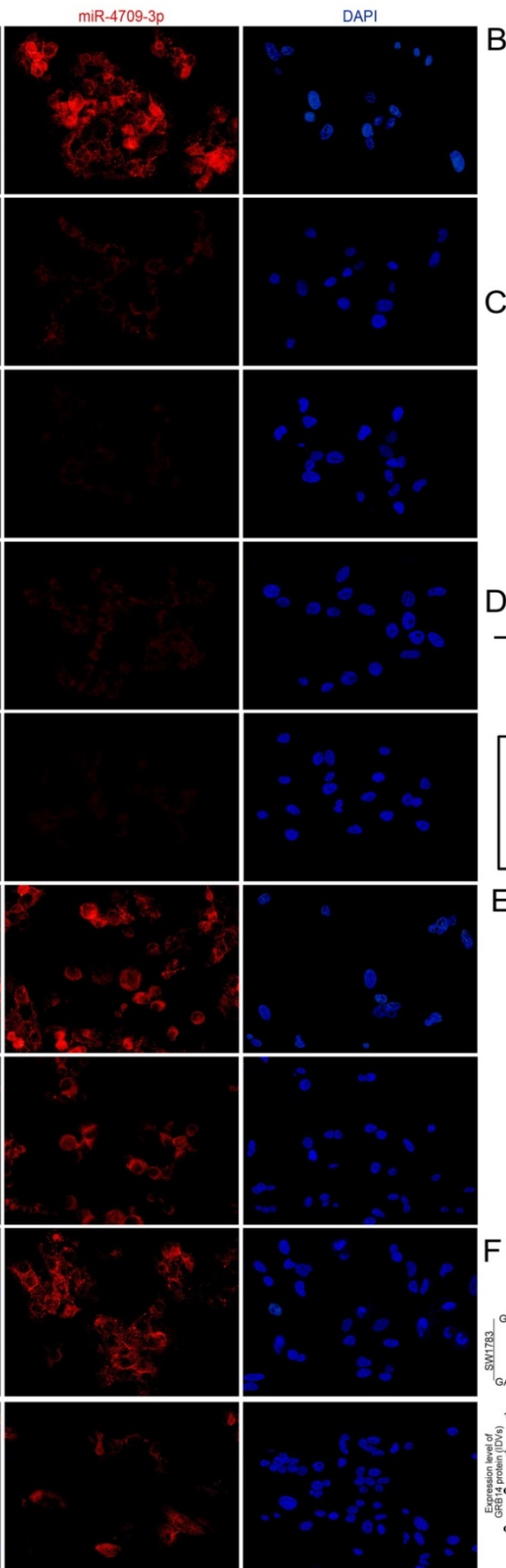

B

C

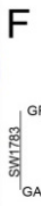

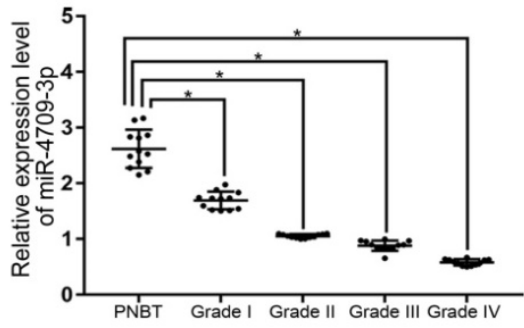
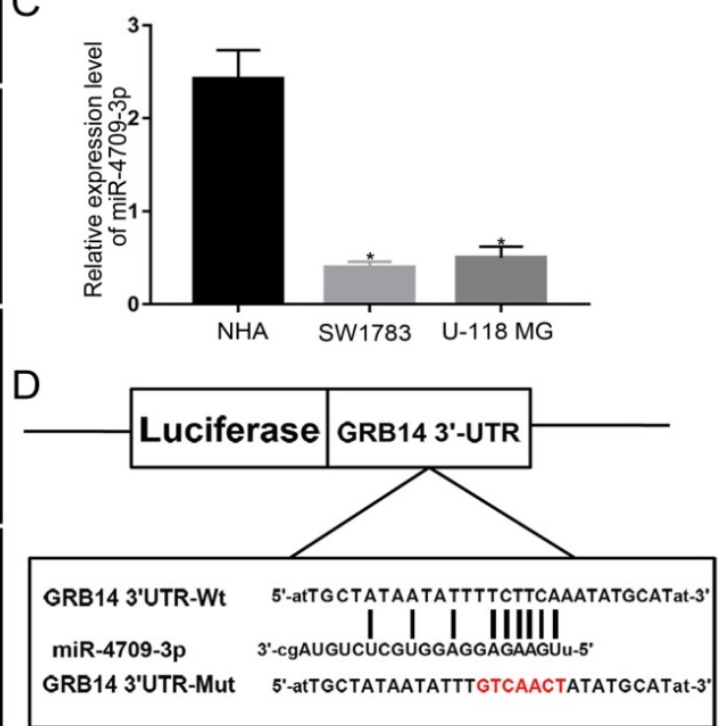

E
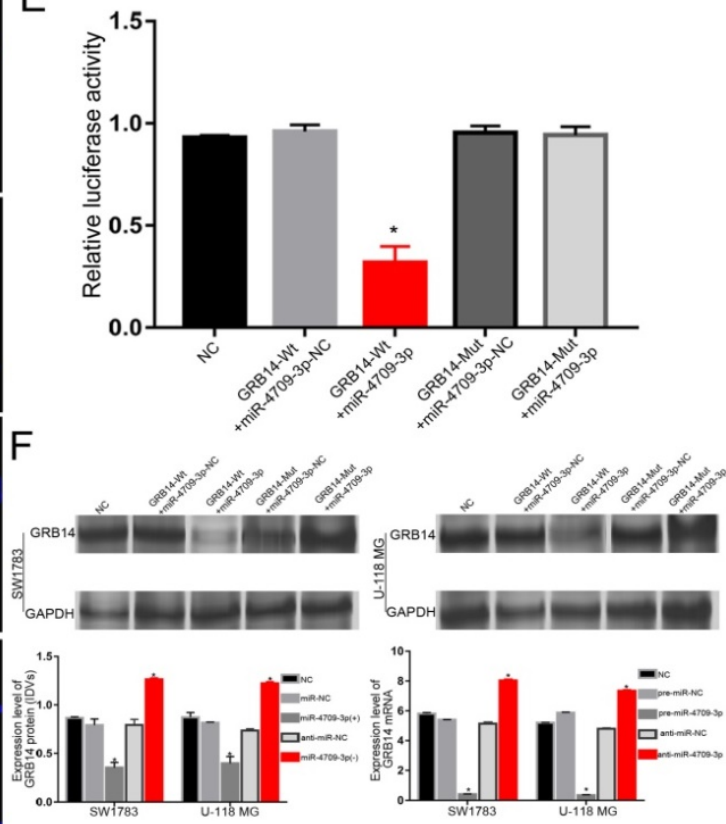

Figure 4. miR-4709-3p was significantly affected by the IdrEXO-transported circ-METRN and exerts its regulatory functions by targeting GRB 14. A. After treatments with IdrEXOs, the levels of miR-4709-3p were significantly impacted in glioblastoma cells with or without the treatment of miR-4709-3p mimics $(n=6)(s c a l e$ bar: 20 $\mu \mathrm{m})$. B. Expression levels of miR-4709-3p in PNBT and glioblastoma $(n=12)$. C. Expression levels of miR-4709-3p in normal human astrocytes $(N H A)$ and glioblastoma cells $(n$ = 6). D. The predicted miR-4709-3p binding sites in GRB14 (GRB14 3'UTR-Wt) and GRB14 3'UTR-Mut. E. Luciferase reporter assay of HEK 293T cells with different co-transfection treatments. F. The expression level of GRB14 protein and mRNA was regulated by miR-4709-3p in glioblastoma cells. $* P<0.05$, $\# P>0.05$. 

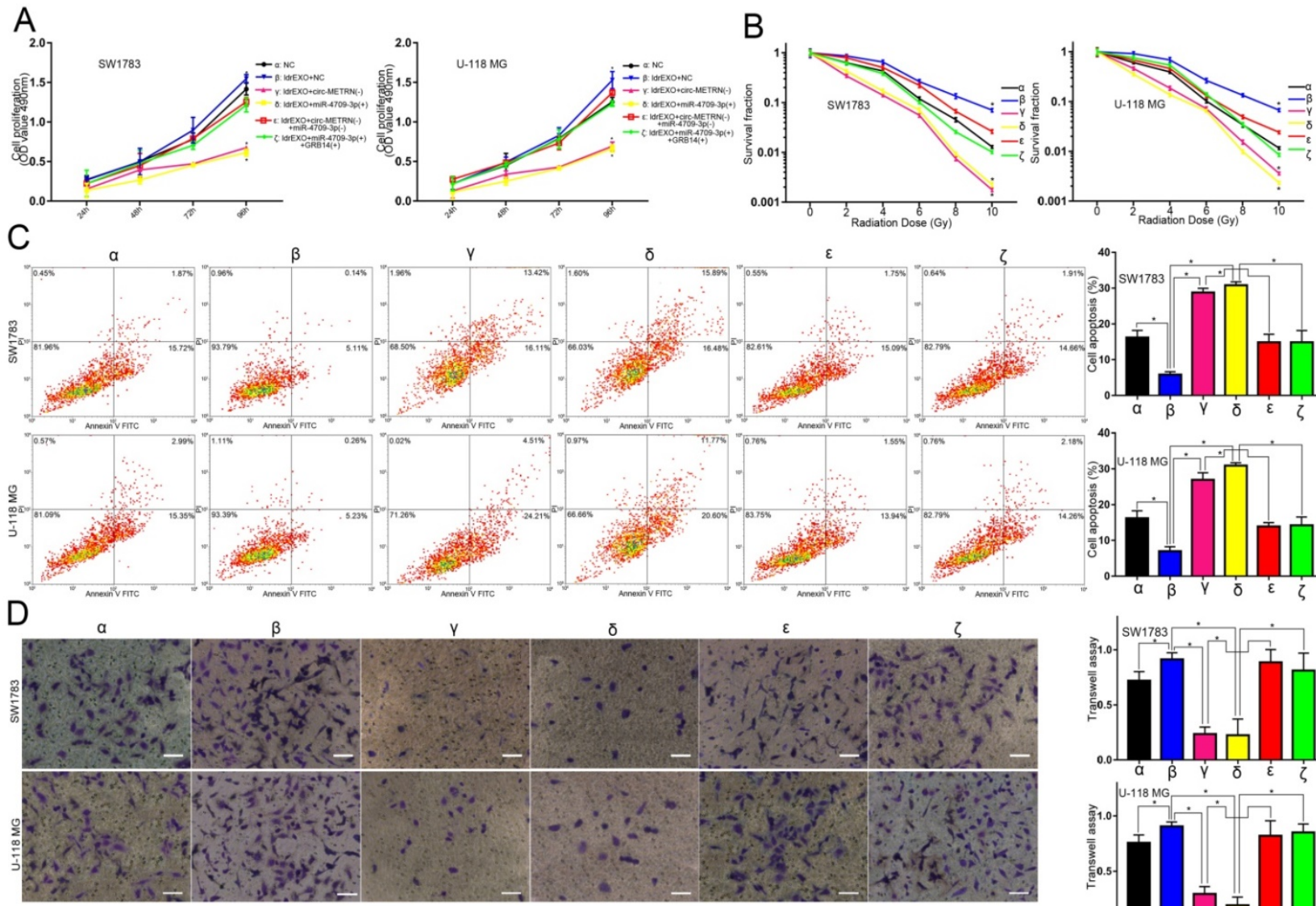

E
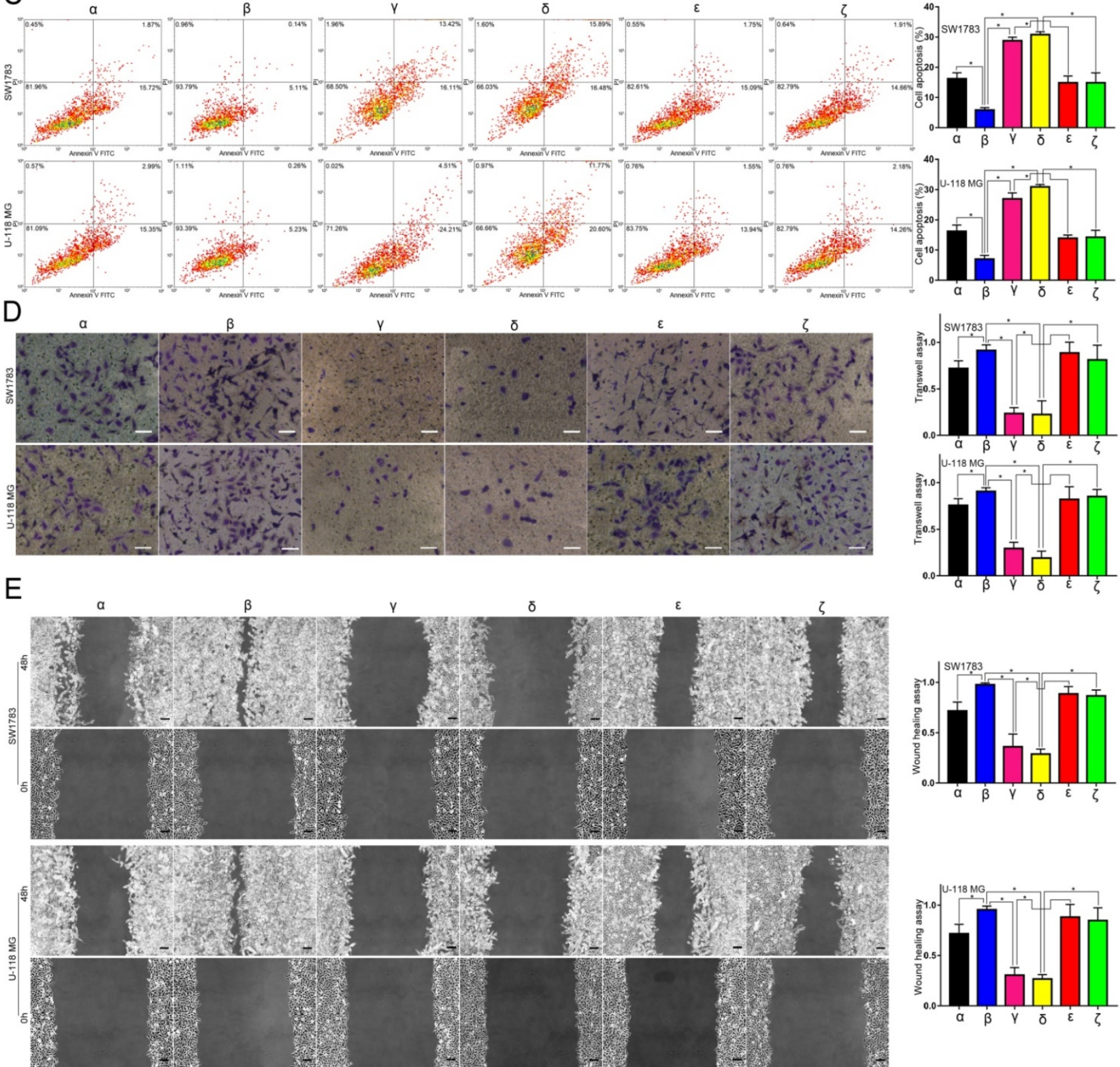

Figure 5. Low-dose radiation-induced exosomal circ-METRN promotes glioblastoma cell progression and radioresistance by regulating the miR-4709-3p/GRB 14 axis. A. Proliferation-promoting effects of circ-METRN-abundant IdrEXOs on glioblastoma cells can be attenuated by miR-4709-3p ( $\delta$ ) and can be rescued by GRB14 (Z). B. Radioresistance-promoting effects of circ-METRN-abundant IdrEXOs on glioblastoma cells can be attenuated by miR-4709-3p ( $\delta$ ) and can be rescued by GRB14 (ל). C. Apoptosis resistance was assessed by flow cytometry with annexin V-FITC/propidium iodide (PI). D-E. Transwell assays and wound healing assays were used to analyze glioblastoma cell migration and invasion ability, respectively. $* P<0.05, \# P>0.05$.

GRB 14 has a regulatory function on PDGFRa. And IdrEXO-derived circ-METRN may have a positive effect on downstream pathways in glioblastoma cells

GRB14 and PDGFRa exhibited high expression levels in glioblastoma tissues and cell lines (Figure
6A-B). There was no significant difference in the expression levels of GRB14 mRNA and protein after knocking down and/or increasing the expression level of PDGFRa (Figure 6C). However, up-regulating GRB14 increased PDGFRa expression level, and down-regulating GRB14 decreased PDGFRa expression level (Figure 6D). 

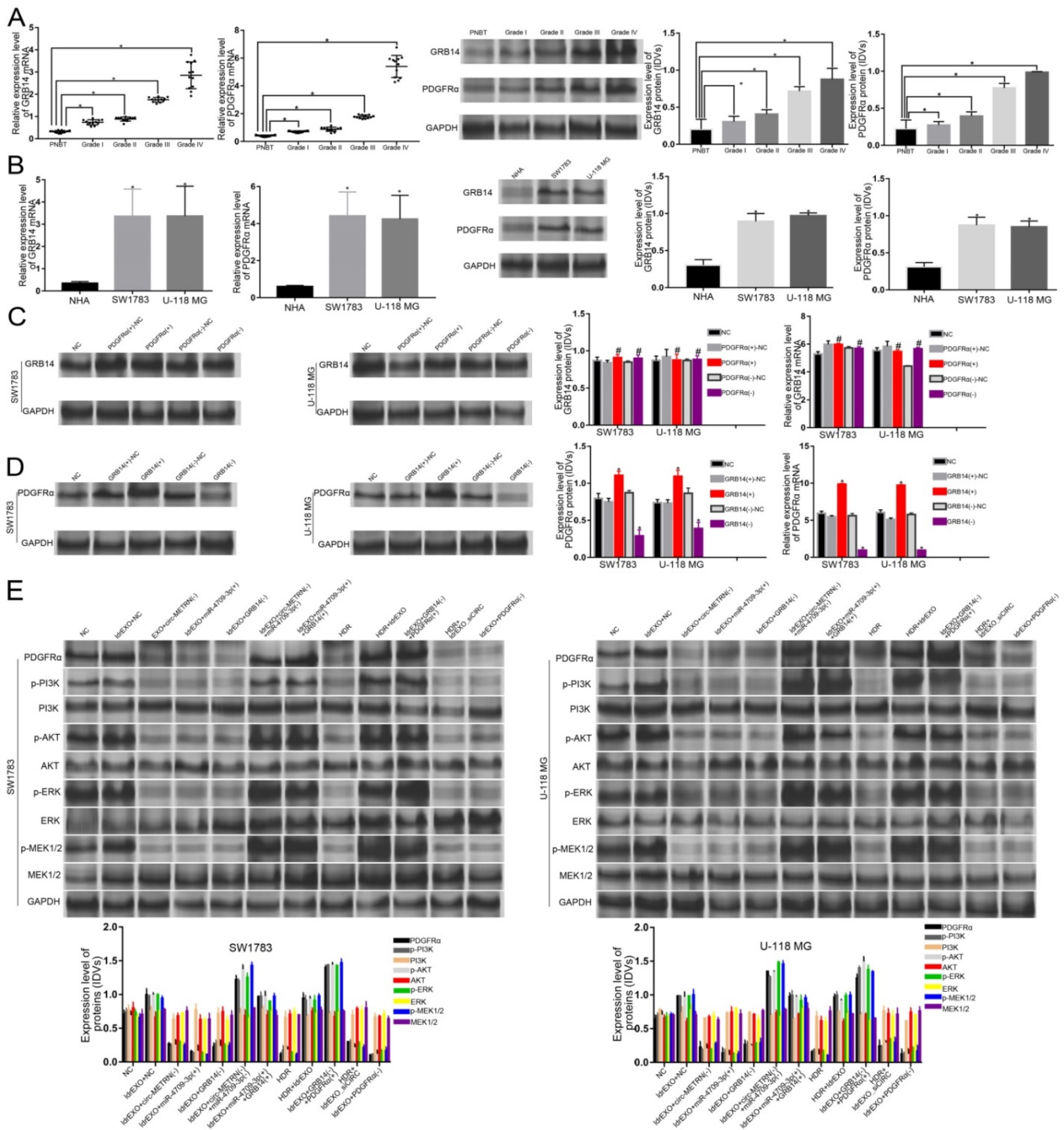

$\mathrm{F}$

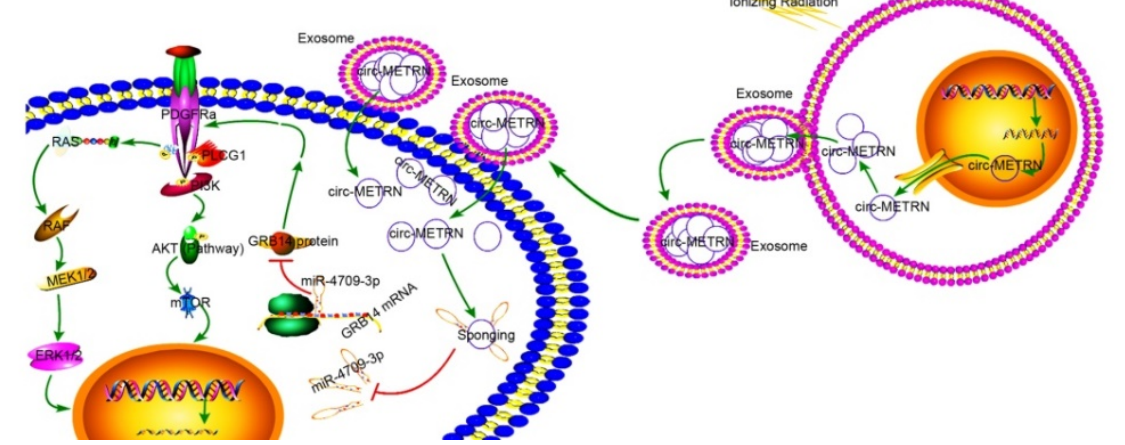

Figure 6. Impacts of IdrEXOs and IdrEXO-derived circ-METRN on the downstream pathway in glioblastoma cells. A. Expression levels of GRB14 and PDGFRa in PNBT and glioblastoma tissues $(n=12)$. B. The relative expression levels of GRB14 and PDGFRa in NHA and glioblastoma cell lines. C. The expression levels of GRB14 protein and mRNA were not affected by PDGFRa in the PDGFRa(+)/PDGFRa(-) group. D. RT-PCR and western-blot analysis further verified that GRB14 can regulate the PDGFRa expression in the GRB14(+)/GRB14(-) group, respectively. E. Western blot analysis showed that PDGFRa, p-PI3K, PI3K, p-AKT, AKT, p-ERK, ERK, p-MEK1/2, and MEK $1 / 2$ were regulated by IdrEXOs, exosomal circ-METRN, and miR-4709-3p/GRB14 axis in SW1783 and U-118MG cells. F. The schematic cartoon of the mechanism of low-dose radiation-induced exosomal circ-METRN via miR-4709-3P/GRB14/PDGFRa pathway in glioblastoma cells was shown. *P<0.05, \#P>0.05. 
The impact of ldrEXOs, exosomal circ-METRN, and miR-4709-3p/GRB14 axis on the proteins of PDGFRa, p-PI3K, PI3K, p-AKT, AKT, p-ERK, ERK, p-MEK1/2, and MEK1/2 were also analyzed by western blot (Figure 6E). The mechanism of low-dose radiation-induced exosomal circ-METRN via miR4709-3p/GRB14/PDGFRa pathway in glioblastoma cells was elucidated by using the schematic cartoon (Figure 6F).

\section{GRB 14 may play glioblastoma-promoting roles through regulating the downstream PDGFRa after treatments with IdrEXOs}

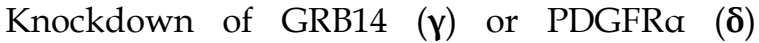
attenuated proliferation, radioresistance, apoptosis radioresistance, invasion, and migration abilities of glioblastoma cell lines previously treated with ldrEXOs (Figure 7A-E). Up-regulation of PDGFRa $(\varepsilon)$ rescued progression and radioresistance of glioblastoma cell lines previously treated with knockdown of GRB14 (Figure 7A-E).

\section{Low-dose radiation-induced exosomal circ- METRN promotes glioblastoma progression and radioresistance in vivo}

The in-vivo experiment showed that treatments with ldrEXOs resulted in the rapid growth of xenografted tumors and a shortened survival period (Figure 8A). HDR treatments reduced tumor growth and prolonged survival period. Moreover, ldrEXOs with high levels of circ-METRN rescued tumor-growth ability of glioblastoma previously treated with HDR while ldrEXOs_siCIRC did not significantly exhibit the rescue effect (Figure 8A).

\section{Low-dose radiation-induced exosomal circ- METRN may be delivered into the bloodstream in the early stages of fractionated radiotherapy}

In this study, detailed information about a total of eighty-four glioblastoma patients were collected (Table 1). Further detection revealed that circMETRN level (Mean \pm SD) in Glioblastoma tissues was $8.499 \pm 0.233$ while circ-METRN levels (Mean \pm SD) in serum exosomes were $1.025 \pm 0.067$ (before operation), $0.995 \pm 0.071$ (before radiotherapy), $2.560 \pm 0.164$ (during the first week of radiotherapy), and $1.737 \pm 0.122$ (after radiotherapy), respectively. The level of serum exosomal circ-METRN detected in the first week of radiotherapy was significantly higher than that before radiotherapy (Figure 8B). These results suggested that high levels of circ-METRN may be delivered into the bloodstream by exosomes in the early stage of fractionated radiotherapy. Compared with the whole course of radiotherapy, the radiation dose delivered in the surgical area of the tumor bed was not high in the early stage of radiotherapy. Serum exosomal circ-METRN detected in the first week of radiotherapy was considered as low-dose radiationinduced exosomal circ-METRN.

High levels of serum exosomal circ-METRN can not only predict a poor prognosis but also assist MRI diagnosis in early detection of recurrence in glioblastoma patients

Survival analysis demonstrated that glioblastoma patients exhibited poor prognosis (1-year OS 75.0\%, 2-year OS 8.3\%; 1-year DFS 2.4\%, 2 -year DFS $0.0 \%$ ). And median OS and DFS were $13.313 \pm 0.085$ months and $8.54 \pm 0.191$ months, respectively. Multivariate analysis revealed that exosomal circ-METRN (detected in the early stage of radiotherapy) was an independent prognostic factor for OS and DFS (Supplementary Material: Table S6). Glioblastoma patients displaying high levels of exosomal circ-METRN exhibited a worse prognosis than other groups (Figure 8C-D). Rapid recurrence after postoperative radiotherapy occurred in patients with high levels of exosomal circ-METRN (detected in the early stage of radiotherapy) (Figure 8D), suggesting significant radiotherapy resistance in these glioblastoma patients. It may be very essential for these patients to seek other more effective and aggressive individualized treatments.

We also explored the diagnostic value of serum exosomal circ-METRN detected in the early stage of radiotherapy. MRI reexamination during radiotherapy indicated abnormal signals in 39 patients (Figure 8E), 22 of whom had high expression of exosomal circ-METRN in the early stage of radiotherapy. And 21 of these 22 patients were subsequently diagnosed by pathology following secondary surgery as recurrence at the site of abnormal signals (Figure 8F). Of the 17 patients with low expression of exosomal circ-METRN in the early stage of radiotherapy, only 3 patients were pathologically diagnosed as having relapses at the abnormal-signal site after secondary surgery. Then, we rediagnosed glioblastoma recurrence using a novel method combining MRI with serum exosomal circ-METRN (detected in the early stage of radiotherapy). According to this combined diagnosis method, cases with both abnormal MR signals and high levels of serum exosomal circ-METRN detected in the early stage of radiotherapy were defined as having very early recurrence (positive); cases with no abnormal MRI signals and with low levels of serum exosomal circ-METRN are not considered to have recurrences (negative) (Supplementary Material: Table S7). Meanwhile, based on pathological 
diagnosis, recurrence on the location of abnormal MRI signals was identified as a positive diagnosis; non-malignant lesion on the location of abnormal MRI signals or recurrence on other locations out of the abnormal-signal area was identified as a negative diagnosis (Supplementary Material: Table S7). According to the analysis, the true positive rate $(0.875$,
$21 / 24)$, true negative rate $(0.960,24 / 25)$, and accuracy $(0.918,45 / 49)$ of this combined diagnosis method were significantly higher than those of MRI diagnosis alone [true positive rate $0.167(4 / 24)$, true negative rate $0.08 \quad(2 / 25)$ and accuracy $0.122 \quad(6 / 49)]$ (Supplementary Material: Table S7).

A
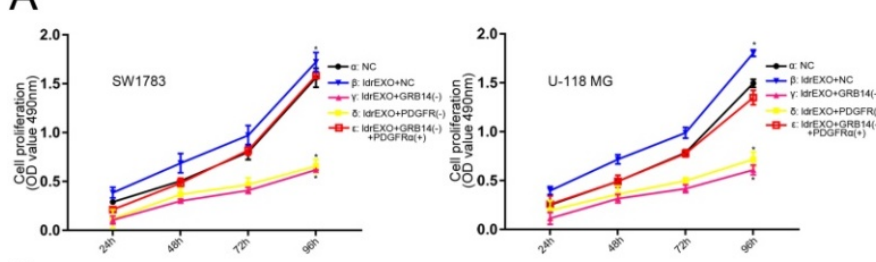

B
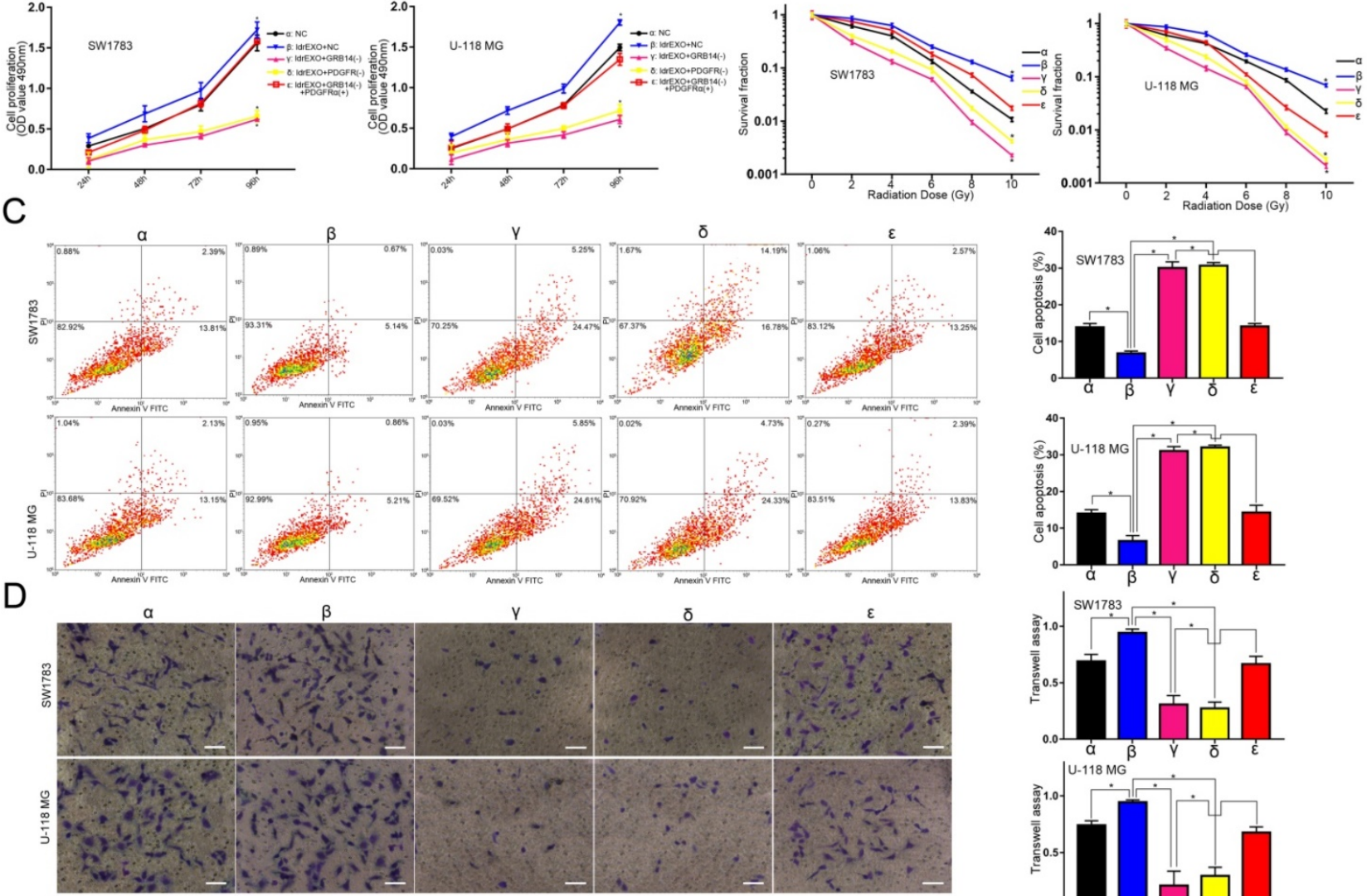

$E$
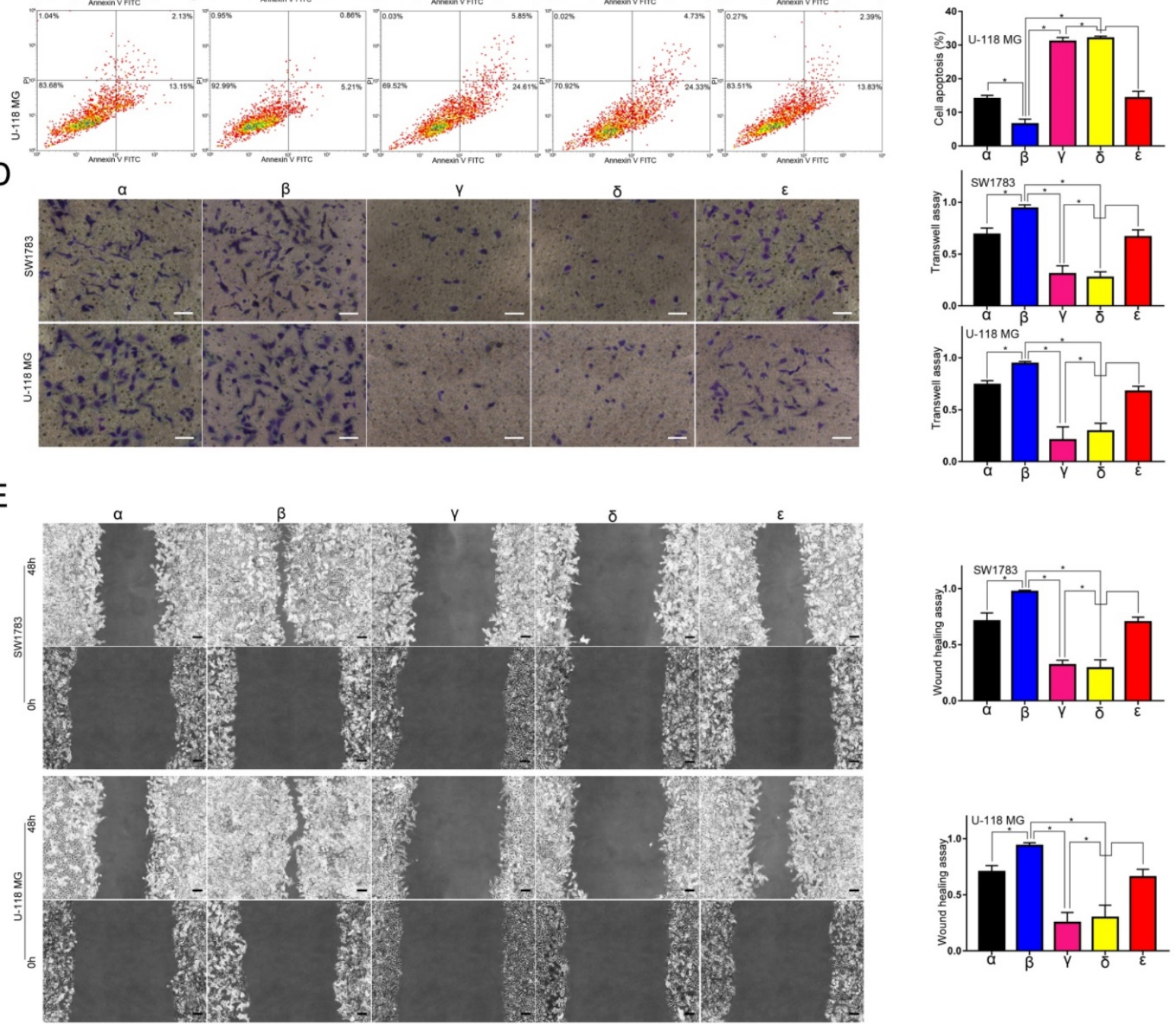

Figure 7. GRB14 may play glioblastoma-promoting roles through regulating the downstream PDGFRa after treatments with IdrEXOs. A. Proliferation-promoting effects of circ-METRN-abundant IdrEXOs on glioblastoma cells can be attenuated by GRB14 (V) inhibition and can be rescued by PDGFRa ( $\varepsilon$ ). B. Radioresistance-promoting effects of circ-METRN-abundant IdrEXOs on glioblastoma cells can be attenuated by GRB14 inhibition ( $\mathrm{Y}$ ) and can be rescued by PDGFR $(\varepsilon)$. C. Apoptosis resistance was assessed by flow cytometry with annexin V-FITC/propidium iodide (PI). D-E. Transwell assays and wound healing assays were used to analyze glioblastoma cell migration and invasion ability, respectively. $* P<0.05, \# P>0.05$. 
A
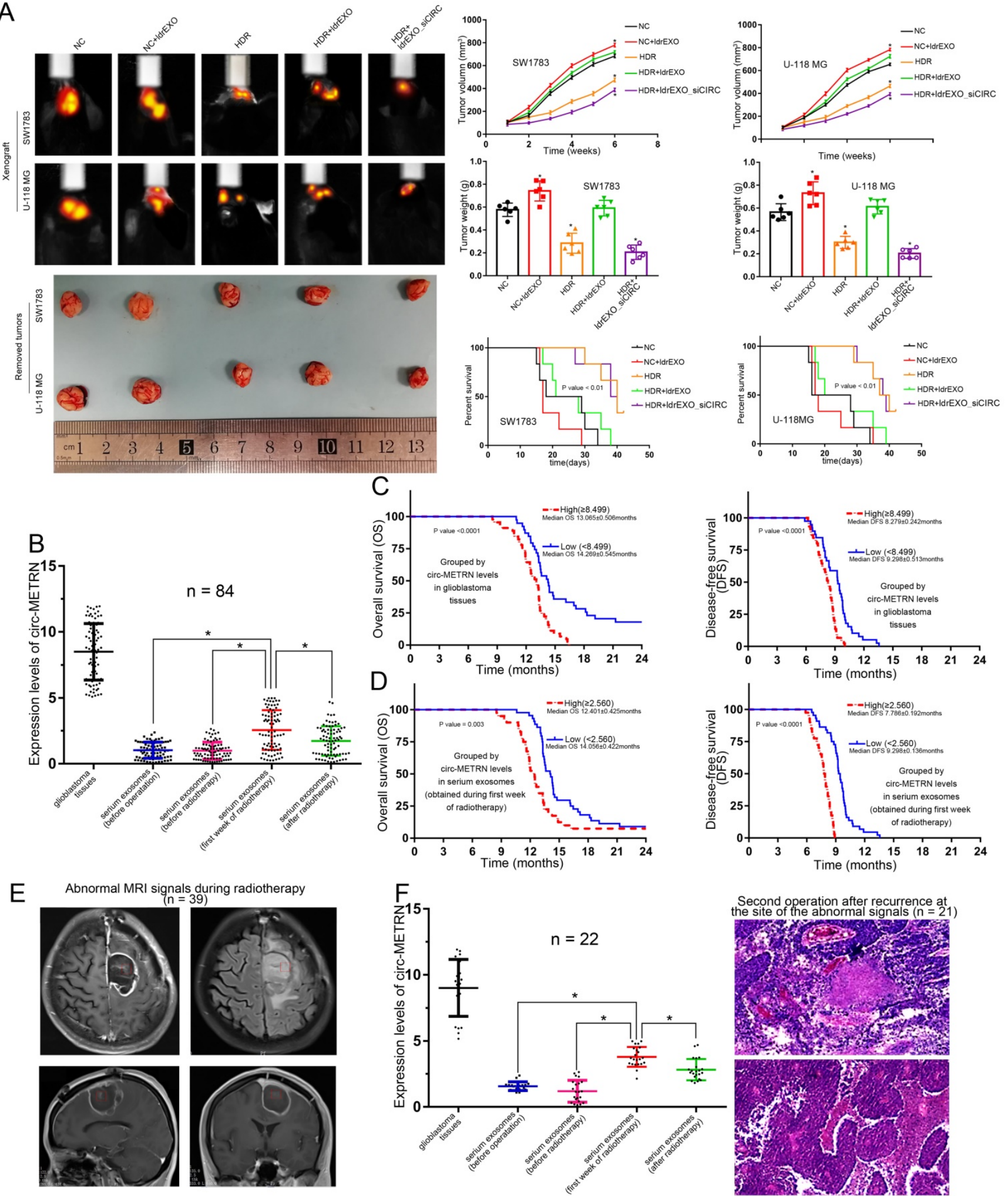

Figure 8. In-vivo experiments on the role of low-dose radiation-induced exosomal circ-METRN and clinical research on its values in glioblastoma progression and radioresistance. A. In vivo fluorescence images of glioblastoma-bearing mice intravenously receiving SW1783 and U-118MG cells with different treatments were obtained before euthanizing mice. Representative pictures of removed xenograft tumors, as well as tumor volume, tumor weight and survival time, are presented ( $\mathrm{n}=6$, each group). B. circ-METRN levels in glioblastoma tissues and serum exosomes (obtained before the first operation, before radiotherapy, during the first week of radiotherapy, or after radiotherapy) were detected and compared. C-D. Exosomal circ-METRN level detected in the early stage of radiotherapy, as well as circ-METRN level in glioblastoma tissue, was an independent prognostic factor affecting OS and DFS. E. MRI reexamination during radiotherapy indicated abnormal signals (red box) in 39 patients. F. 21 of these 22 patients with high levels of circ-METRN and abnormal MRI signals during radiotherapy were then identified pathologically as recurrence at the site of abnormal signals. $* P<0.05$, $\# P>0.05$. 
Table 1. Clinical characteristics of glioblastoma patients at baseline

\begin{tabular}{|c|c|c|c|c|c|c|}
\hline \multirow[t]{3}{*}{ Variable } & \multicolumn{6}{|c|}{ Characteristics } \\
\hline & \multicolumn{3}{|c|}{$\begin{array}{l}\text { circ-METRM in } \\
\text { glioblastoma tissue }\end{array}$} & \multicolumn{3}{|c|}{$\begin{array}{l}\text { circ-METRN in SE (first } \\
\text { week of radiotherapy) }\end{array}$} \\
\hline & $\begin{array}{l}\text { Low } \\
(<8.499)\end{array}$ & $\begin{array}{l}\text { High } \\
(\geq 8.499)\end{array}$ & $\begin{array}{l}P \\
\text { value }\end{array}$ & $\begin{array}{l}\text { Low } \\
(<2.560)\end{array}$ & $\begin{array}{l}\text { High } \\
(\geq 2.560)\end{array}$ & $\begin{array}{l}P \\
\text { value }\end{array}$ \\
\hline \multicolumn{7}{|c|}{ (2) } \\
\hline$<50$ & 19 & 22 & 0.988 & 20 & 21 & 0.525 \\
\hline$\geq 50$ & 20 & 23 & & 24 & 19 & \\
\hline \multicolumn{7}{|l|}{ Gender } \\
\hline Male & 19 & 20 & 0.700 & 18 & 21 & 0.293 \\
\hline Female & 20 & 25 & & 26 & 19 & \\
\hline \multicolumn{7}{|c|}{ Performance status score (ECOG) } \\
\hline $0-2$ & 22 & 27 & 0.743 & 25 & 24 & 0.771 \\
\hline$>2$ & 17 & 18 & & 19 & 16 & \\
\hline \multicolumn{7}{|c|}{ Location of the tumor } \\
\hline Right & 9 & 14 & 0.188 & 13 & 10 & 0.307 \\
\hline Left & 15 & 20 & & 20 & 15 & \\
\hline $\begin{array}{l}\text { Median and/or } \\
\text { bilateral }\end{array}$ & 15 & 11 & & 11 & 15 & \\
\hline \multicolumn{7}{|l|}{ Multifocal tumor } \\
\hline Yes & 4 & 3 & 0.558 & 5 & 2 & 0.298 \\
\hline No & 35 & 42 & & 39 & 38 & \\
\hline \multicolumn{7}{|l|}{ Signs and symptoms } \\
\hline Epilepsy & 10 & 5 & 0.292 & 10 & 5 & 0.016 \\
\hline Headache & 4 & 8 & & 10 & 2 & \\
\hline $\begin{array}{l}\text { High intracranial } \\
\text { pressure }\end{array}$ & 4 & 8 & & 6 & 6 & \\
\hline $\begin{array}{l}\text { Mental status } \\
\text { disorders }\end{array}$ & 11 & 7 & & 7 & 11 & \\
\hline Sensory-motor deicit & 5 & 8 & & 6 & 7 & \\
\hline Other & 5 & 9 & & 5 & 9 & \\
\hline \multicolumn{7}{|c|}{ Tumor molecular profile } \\
\hline IDH wild type & 36 & 42 & 0.858 & 38 & 40 & 0.015 \\
\hline IDH mutated type & 3 & 3 & & 6 & 0 & \\
\hline MGMT methylated & 5 & 4 & 0.567 & 6 & 3 & 0.370 \\
\hline MGMT unmethylated & 34 & 41 & & 38 & 37 & \\
\hline \multicolumn{7}{|c|}{ Postoperative therapy } \\
\hline Radiotherapy & 30 & 35 & 0.876 & 31 & 34 & 0.101 \\
\hline $\begin{array}{l}\text { Radiotherapy+ } \\
\text { systematic treatments }\end{array}$ & 9 & 10 & & 13 & 6 & \\
\hline \multicolumn{7}{|c|}{ Abnormal MRI signals during radiotherapy } \\
\hline Yes & 15 & 24 & 0.136 & 17 & 22 & 0.177 \\
\hline No & 24 & 21 & & 27 & 18 & \\
\hline
\end{tabular}

SE: serum exosome; ECOG: Eastern Cooperative Oncology Group; MGMT: methylguanine methyltransferase; IDH: isocitrate dehydrogenase; SD: standard deviation; MRI: Magnetic resonance imaging.

\section{Discussion}

The high mortality rate of glioblastoma is closely related to the recurrence of this malignant tumor after postoperative radiochemotherapy [1]. The advancement of radiotherapy technology has neither reduced the recurrence rate of glioblastoma nor has it effectively changed the radioresistance of glioblastoma cells, especially for MGMTunmethylated patients $[2,3,5]$. Previous molecular biology studies have also shown that glioblastoma may progress through multiple signaling pathways $[13,14]$. Exosomes and their contents play an important role in glioblastoma progression and therapeutic resistance while ionizing radiation also played a positive role in the secretion of exosomes [24-26].

In this study, exosomes induced by low-dose radiation (ldrEXOs) were identified as an important factor that impacted glioblastoma progression and radioresistance. ldrEXOs promoted the expression of $\mathrm{Y}-\mathrm{H} 2 \mathrm{AX}$ which has a key role in DNA double-strand break repair in glioblastoma radioresistance [32]. This result was similar to that of previous research by Qiupeng Zheng et al [22]. They noted that exosomes derived from AHIF-overexpressing glioblastoma cells promoted viability, invasion, and radioresistance [22]. However, the study of Farias et al. demonstrated that some sorts of exosomes derived from special sources can enhance radiotherapy-induced cell death in malignant tumors [28]. And the regulatory function of exosomes derived from mesenchymal stem cells is quite different from that of ldrEXOs [28].

Moreover, the content of exosomes induced by radiation needs to be further studied, which is one of the reasons why the function of radiation-induced exosomes is controversial [15]. This study identified for the first time that ldrEXOs are enriched with circRNAs and high-dose radiation did not significantly promote the secretion of exosomal circRNAs. It implied that the regulatory function of ldrEXOs in glioblastoma may depend on exosomal circRNAs. In the present study, ldrEXOs-derived circ-METRN played a key role in the tumor progression and radioresistance while ldrEXOs with knockdown of circ-METRN did not exhibit a similar tumor-promoting function. This effect of exosomal circ-METRN is similar to that in the research of Ding et al [23]. In their research, exosomal circNFIX was up-regulated in the serum of TMZ-resistant cases [23]. Exosomal circNFIX from TMZ-resistant cells conferred TMZ resistance to recipient sensitive cells through promoting cell migration and invasion and suppressing cell apoptosis under TMZ exposure [23]. Han et al. also found that Circ-HIPK3 was increased in TMZ-resistant malignant glioma cells and their exosomes [33]. Furthermore, circRNA has been identified as a "miRNA sponge" that functions as a miRNA inhibitor [14]. This circRNA-miRNA regulatory network affects target genes and ultimately regulates malignant progression [14]. In other malignant tumors, the tumor-promoting effects of exosomal circRNAs can be observed. Exosomal circRNA PDE8A promotes pancreatic cancer invasion via the miR-338/MACC1/MET pathway [16]. Exosomal circRNA-100338 enhances invasiveness, metastasis, and angiogenesis in hepatocellular carcinoma [17]. Gastric cancer-derived exosomal circRNA ciRS-133 promotes white adipose browning by targeting the miR-133/PRDM16 pathway [18]. 
Exosomal circPACRGL promotes colorectal cancer progression via the miR-142-3p/miR-506-3p/TGF- $\beta 1$ axis [19]. Exosome circ_0044516 promotes prostate cancer cell proliferation and metastasis as a potential biomarker via miR-29a-3p [21]. In the present study, this sponging relationship was confirmed between circ-METRN and miR-4709-3p. This circ-METRNmiR-4709-3p network had a significant regulatory effect on the progression of glioblastoma, which is consistent with the results of previous studies [14]. The promoting effect of ldrEXOs on glioblastoma progression and radioresistance can be decreased by treatments of miR-4709-3p mimics, suggesting that miR-4709-3p may play a tumor-suppressing role in glioblastoma. Therefore, the regulatory function of ldrEXOs in glioblastoma may depend on exosomal circ-METRN, and exosomal circ-METRN exerts its regulatory functions in promoting glioblastoma progression and radioresistance through sponging miR-4709-3p.

Besides, GRB14 was determined as a direct target of miR-4709-3p, and miR-4709-3p plays a further role in glioblastoma cell inhibition by targeting GRB14. In this study, increasing the expression level of GRB14 can rescue the proliferation, migration, and invasion of glioblastoma cells, despite the different expression levels of miR-4709-3p in different groups. This coincides with the previous studies that GRB14 could promote tumor progression [34, 35]. And GRB14 is highly expressed in malignant tumor cells and was regarded as a predictor of poor prognosis in tumor patients [34, 35]. However, the expression level, role, and prognostic value of GRB14 in malignant tumors had often been controversial [34-37]. Some other studies suggested that GRB14 was down-regulated in malignant tumor cells and can be used as a marker for good prognosis in cancer patients [36, 37]. Detailed analysis of these previous studies revealed that the controvery may origin in the different binding pattern of GRB14 domains to growth factor receptors [38]. In fact, the functional GRB14 binded to different growth factors with its various domains [38]. For instance, GRB14 can bind to some growth factor receptors, including PDGFR, through its SH2 domain [38]. Differently, GRB14 can bind to insulin receptors mainly through small domains (BPS or PIR domains) adjacent to the SH2 domain [38]. Thus, GRB14 can bind to different growth factor receptors through different domains, and different binding patterns may lead to different effects [38].

The mechanism that GRB14 promoted tumor progression may be related to the downstream signal transduction pathway PDGFR. In cells, GRB14 can form a fusion protein with glutathione S-transferase, which had a strong binding ability with PDGFR, while the expression of GRB14 is not affected by the binding mode of PDGFR-GRB14 [39]. In this present study, the expression of PDGFRa was impacted by GRB14. Inhibition of GRB14 attenuated the tumor-promoting function of ldrEXOs although circ-METRN was highly expressed in glioblastoma cell lines previously treated with ldrEXOs. Up-regulating PDGFRa can recover the tumor-promoting function of ldrEXOs in glioblastoma cell lines previously treated with inhibition of GRB14. These results indicated that PDGFRa may be located downstream of the signal transduction pathway and may also underscore a critical role in glioblastoma progression and radioresistance. This is consistent with previous studies on the carcinogenic effects of PDGFR [40-43]. H Feng et al. also determined that PDGFRa can promote the progression of glioblastoma by regulating the expression of Akt, Erk1/2, and other genes in the glioblastoma-related signal transduction pathway [40]. Similar results were found in our previous research [40]. Taken together, low-dose radiation-induced exosomal circ-METRN may exert its positive regulatory functions in glioblastoma progression and radioresistance via miR-4709-3p/ GRB14/PDGFRa pathway.

We further explored the clinical value of low-dose radiation-induced exosomal circ-METRN (detected in the early stage of radiotherapy). This study provides strong evidence for the identification of early and rapid recurrence of glioblastoma. In the early stage of radiotherapy, low-dose radiation may promote the release and entry of exosomes into the bloodstream, thus affecting the progression and radioresistance of glioblastoma, and finally leading to the recurrence. This tumor-promoting effect of low-dose radiation-induced exosomal circ-METRN enables it to be an effective biomarker and a therapeutic target for glioblastoma patients with poor prognosis. Meanwhile, serum exosomal circ-METRN can also assist MRI diagnosis in the early detection of recurrence in glioblastoma patients. In the very early progression, the specificity of MRI diagnosis is limited because the postoperative inflammation, brain edema, and pseudoprogression can all appear as slight abnormal signals on MRI $[4,6]$. Our study revealed a novel diagnosis method that combined MRI and serum exosomal circ-METRN levels detected in the early stage of radiotherapy. This combined diagnosis method of abnormal signals improves the accuracy of MRI diagnosis in detecting the very early recurrence of glioblastoma. Therefore, low-dose radiation-induced exosomal circ-METRN (detected in the early stage of radiotherapy) has important clinical values. For patients who display high levels of exosomal circ-METRN (detected in the first week of 
radiotherapy) or who suffer a very early recurrence, it may be essential to seek other more effective and aggressive individualized treatments.

In summary, the present study is the first to examine the circ-METRN levels in low-dose radiation-induced exosomes and to reveal the role of exosomal circ-METRN via miR-4709-3p/GRB14/ PDGFRa pathway in understanding the pathogenesis of glioblastoma, providing novel insights for identifying new biomarkers or potential theranostic targets.

\section{Abbreviations}

ldrEXOs: low-dose radiation-induced exosome; LDR: low-dose radiation; HDR: high-dose radiation; ldrEXOs_siCIRC: exosome derived from cells treated with knockdown of circ-METRN; circ-METRN: the circular METRN RNA; linear-METRN: the linear METRN mRNA; circRNAs: circular RNAs; miRNAs: microRNAs; METRN: meteorin, glial cell differentiation regulator; PDGFRa: Platelet-derived growth factor receptor alpha; GRB: growth factor receptor-bound protein; PNBT: peritumoral normal brain tissue; NC: negative control; NHA: normal human astrocytes; DAPI: 4, 6-diamidino-2-phenylindole; siRNA: small interfering RNA; FISH: fluorescence in situ hybridization; RT-PCR: quantitative real-time PCR; MTT: 3-(4,5-dimethyl-2thiazolyl)-2,5-diphenyl-2-H-tetrazolium bromide; HEK: human embryonic kidney; SD: standard deviation; OS: overall survival; DFS: disease-free survival.

\section{Supplementary Material}

Supplementary tables.

http://www.ijbs.com/v17p1061s1.pdf

\section{Acknowledgements}

This study was supported by the Scientific Research Initiation Foundation for Youth of the First Affiliated Hospital of Zhengzhou University (No.161032). We thank Guan Zhai (Neurosurgery Department of the First Affiliated Hospital of Zhengzhou University), Jianbo Gao (Medical Imaging Department of the First Affiliated Hospital of Zhengzhou University), and Wenchai Li (Pathology Department of the First Affiliated Hospital of Zhengzhou University) for providing professional knowledge and technical supports.

\section{Competing Interests}

The authors have declared that no competing interest exists.

\section{References}

1. Ostrom QT, Gittleman H, de Blank PM, Finlay JL, Gurney JG, McKeanCowdin R, et al. American Brain Tumor Association Adolescent and Young Adult Primary Brain and Central Nervous System Tumors Diagnosed in the United States in 2008-2012. Neuro Oncol. 2016; 18 Suppl 1: i1-50.

2. Lee DY, Chunta JL, Park SS, Huang J, Martinez AA, Grills IS, et al. Pulsed versus conventional radiation therapy in combination with temozolomide in a murine orthotopic model of glioblastoma multiforme. Int J Radiat Oncol Biol Phys. 2013; 86: 978-85.

3. Beauchesne P. Three-times daily ultrafractionated radiation therapy, a novel and promising regimen for glioblastoma patients. Cancers (Basel). 2013; 5: 1199-211.

4. Kebir S, Schmidt T, Weber M, Lazaridis L, Galldiks N, Langen KJ, et al. A Preliminary Study on Machine Learning-Based Evaluation of Static and Dynamic FET-PET for the Detection of Pseudoprogression in Patients with IDH-Wildtype Glioblastoma. Cancers (Basel). 2020; 12:

5. Fogh SE, Andrews DW, Glass J, Curran W, Glass C, Champ C, et al. Hypofractionated stereotactic radiation therapy: an effective therapy for recurrent high-grade gliomas. J Clin Oncol. 2010; 28: 3048-53.

6. Elshafeey N, Kotrotsou A, Hassan A, Elshafei N, Hassan I, Ahmed S, et al. Multicenter study demonstrates radiomic features derived from magnetic resonance perfusion images identify pseudoprogression in glioblastoma. Nat Commun. 2019; 10: 3170

7. van den Berg J, Castricum K, Meel MH, Goedegebuure R, Lagerwaard FJ, Slotman BJ, et al. Development of transient radioresistance during fractionated irradiation in vitro. Radiother Oncol. 2020; 148: 107-14.

8. Short SC, Mitchell SA, Boulton P, Woodcock M, Joiner MC. The response of human glioma cell lines to low-dose radiation exposure. Int J Radiat Biol. 1999; 75: 1341-8.

9. Leder K, Pitter K, LaPlant Q, Hambardzumyan D, Ross BD, Chan TA, et al. Mathematical modeling of PDGF-driven glioblastoma reveals optimized radiation dosing schedules. Cell. 2014; 156: 603-16.

10. Short SC, Kelly J, Mayes CR, Woodcock M, Joiner MC. Low-dose hypersensitivity after fractionated low-dose irradiation in vitro. Int J Radiat Biol. 2001; 77: 655-64.

11. Camphausen K, Moses MA, Beecken WD, Khan MK, Folkman J, O'Reilly MS. Radiation therapy to a primary tumor accelerates metastatic growth in mice. Cancer Res. 2001; 61: 2207-11.

12. Zhou YC, Liu JY, Li J, Zhang J, Xu YQ, Zhang HW, et al. Ionizing radiation promotes migration and invasion of cancer cells through transforming growth factor-beta-mediated epithelial-mesenchymal transition. Int J Radiat Oncol Biol Phys. 2011; 81: 1530-7.

13. Cao Q, Wang X, Shi Y, Zhang M, Yang J, Dong M, et al. FOXC1 silencing inhibits the epithelial-to-mesenchymal transition of glioma cells: Involvement of $\beta$-catenin signaling. Mol Med Rep. 2019; 19: 251-61.

14. Cao $Q$, Shi $Y$, Wang $X$, Yang J, Mi Y, Zhai G, et al. Circular METRN RNA hsa_circ_0037251 Promotes Glioma Progression by Sponging miR-1229-3p and Regulating mTOR Expression. Sci Rep. 2019; 9: 19791.

15. Ni J, Bucci J, Malouf D, Knox M, Graham P, Li Y. Exosomes in Cancer Radioresistance. Front Oncol. 2019; 9: 869.

16. Li Z, Yanfang W, Li J, Jiang P, Peng T, Chen K, et al. Tumor-released exosomal circular RNA PDE8A promotes invasive growth via the miR-338/MACC1/ MET pathway in pancreatic cancer. Cancer Lett. 2018; 432: 237-50.

17. Huang $X Y$, Huang $Z L$, Huang J, $X u B$, Huang $X Y, X u$ YH, et al. Exosomal circRNA-100338 promotes hepatocellular carcinoma metastasis via enhancing invasiveness and angiogenesis. J Exp Clin Cancer Res. 2020; 39: 20.

18. Zhang H, Zhu L, Bai M, Liu Y, Zhan Y, Deng T, et al. Exosomal circRNA derived from gastric tumor promotes white adipose browning by targeting the miR-133/PRDM16 pathway. Int J Cancer. 2019; 144: 2501-15.

19. Shang A, Gu C, Wang W, Wang X, Sun J, Zeng B, et al. Exosomal circPACRGL promotes progression of colorectal cancer via the miR-142-3p/miR-506-3pTGF- $\beta 1$ axis. Mol Cancer. 2020; 19: 117

20. Boelens MC, Wu TJ, Nabet BY, Xu B, Oiu Y, Yoon T, et al. Exosome transfer from stromal to breast cancer cells regulates therapy resistance pathways. Cell. 2014; 159: 499-513.

21. Li T, Sun X, Chen L. Exosome circ 0044516 promotes prostate cancer cell proliferation and metastasis as a potential biomarker. J Cell Biochem. 2020; 121: $2118-26$

22. Dai X, Liao K, Zhuang Z, Chen B, Zhou Z, Zhou S, et al. AHIF promotes glioblastoma progression and radioresistance via exosomes. Int J Oncol. 2019; 54: 261-70

23. Ding C, Yi X, Wu X, Bu X, Wang D, Wu Z, et al. Exosome-mediated transfer of circRNA CircNFIX enhances temozolomide resistance in glioma. Cancer Lett. 2020; 479: 1-12.

24. Jabbari N, Nawaz M, Rezaie J. Ionizing Radiation Increases the Activity of Exosomal Secretory Pathway in MCF-7 Human Breast Cancer Cells: A possible way to communicate resistance against Radiotherapy. Int J Mol Sci. 2019; 20. DOI: 10.3390/ijms20153649.

25. Mutschelknaus L, Azimzadeh O, Heider T, Winkler K, Vetter M, Kell R, et al. Radiation alters the cargo of exosomes released from squamous head and neck cancer cells to promote migration of recipient cells. Sci Rep. 2017; 7: 12423.

26. Hazawa M, Tomiyama K, Saotome-Nakamura A, Obara C, Yasuda T, Gotoh T, et al. Radiation increases the cellular uptake of exosomes through CD29/CD81 complex formation. Biochem Biophys Res Commun. 2014; 446: 1165-71. 
27. Min H, Sun $X$, Yang $X$, Zhu H, Liu J, Wang Y, et al. Exosomes Derived from Irradiated Esophageal Carcinoma-Infiltrating T Cells Promote Metastasis by Inducing the Epithelial-Mesenchymal Transition in Esophageal Cancer Cells. Pathol Oncol Res. 2018; 24: 11-8.

28. de Araujo FV, O'Valle F, Serrano-Saenz S, Anderson P, Andrés E, LópezPeñalver J, et al. Exosomes derived from mesenchymal stem cells enhance radiotherapy-induced cell death in tumor and metastatic tumor foci. Mol Cancer. 2018; 17: 122

29. Guan Y, Cao Z, Du J, Liu T, Wang T. Circular RNA circPITX1 knockdown inhibits glycolysis to enhance radiosensitivity of glioma cells by miR-329-3p/ NEK2 axis. Cancer Cell Int. 2020; 20: 80.

30. Liu J, Xue N, Guo Y, Niu K, Gao L, Zhang S, et al. CircRNA_100367 regulated the radiation sensitivity of esophageal squamous cell carcinomas through miR-217/Wnt3 pathway. Aging (Albany NY). 2019; 11: 12412-27.

31. Chen W, Quan Y, Fan S, Wang H, Liang J, Huang L, et al. Exosometransmitted circular RNA hsa_circ_0051443 suppresses hepatocellular carcinoma progression. Cancer Lett. 2020; 475: 119-28.

32. Kowalski-Chauvel A, Modesto A, Gouaze-Andersson V, Baricault L, Gilhodes J, Delmas C, et al. Alpha-6 integrin promotes radioresistance of glioblastoma by modulating DNA damage response and the transcription factor Zeb1. Cell Death Dis. 2018; 9: 872

33. Han C, Wang S, Wang H, Zhang J. Exosomal Circ-HIPK3 Facilitates Tumor Progression and Temozolomide Resistance by Regulating miR-421/ZIC5 Axis in Glioma. Cancer Biother Radiopharm. 2020. DOI: 10.1089/cbr.2019.3492.

34. Balogh K, Asa SL, Zheng L, Cassol C, Cheng S, Ezzat S. The insulin resistance Grb14 adaptor protein promotes thyroid cancer ret signaling and progression. Oncogene. 2012; 31: 4012-21.

35. Yang P, Wei J, Li W, He F, Zeng S, Zhang T, et al. High expression of growth factor receptor-bound protein 14 predicts poor prognosis for colorectal cancer patients. Biotechnol Lett. 2016; 38: 1043-7.

36. Huang $\mathrm{O}$, Jiang $\mathrm{M}$, Zhang $\mathrm{X}$, Xie $\mathrm{Z}$, Chen $\mathrm{X}, \mathrm{Wu}$ J, et al. Grb14 as an independent good prognosis factor for breast cancer patients treated with neoadjuvant chemotherapy. Jpn J Clin Oncol. 2013; 43: 1064-72.

37. Morzyglod L, Caüzac M, Popineau L, Denechaud PD, Fajas L, Ragazzon B, et al. Growth factor receptor binding protein 14 inhibition triggers insulininduced mouse hepatocyte proliferation and is associated with hepatocellular carcinoma. Hepatology. 2017; 65: 1352-68.

38. Scharf PJ, Witney J, Daly R, Lyons BA. Solution structure of the human Grb14-SH2 domain and comparison with the structures of the human Grb7$\mathrm{SH} 2$ /erbB2 peptide complex and human Grb10-SH2 domain. Protein Sci. 2004; 13: 2541-6.

39. Daly RJ, Sanderson GM, Janes PW, Sutherland RL. Cloning and characterization of GRB14, a novel member of the GRB7 gene family. J Biol Chem. 1996; 271: 12502-10.

40. Feng H, Liu KW, Guo P, Zhang P, Cheng T, McNiven MA, et al. Dynamin 2 mediates PDGFR $\alpha$-SHP-2-promoted glioblastoma growth and invasion. Oncogene. 2012; 31: 2691-702.

41. Zhang L, Zhang W, Li Y, Alvarez A, Li Z, Wang Y, et al. SHP-2-upregulated ZEB1 is important for PDGFR $\alpha$-driven glioma epithelial-mesenchymal transition and invasion in mice and humans. Oncogene. 2016; 35: 5641-52.

42. Xu R, Ji J, Zhang X, Han M, Zhang C, Xu Y, et al. PDGFA/PDGFR $\alpha$-regulated GOLM1 promotes human glioma progression through activation of AKT. J Exp Clin Cancer Res. 2017; 36: 193.

43. Sang Y, Hou Y, Cheng R, Zheng L, Alvarez AA, Hu B, et al. Targeting PDGFR $\alpha$-activated glioblastoma through specific inhibition of SHP-2mediated signaling. Neuro Oncol. 2019; 21: 1423-35. 